\title{
Predicting mechanical property plateau in laser polymer powder bed fusion additive manufacturing via the critical coalescence ratio
}

\author{
Camden A. Chatham ${ }^{\text {a,b,e }}$, Michael J. Bortner ${ }^{\text {a,c }}$, Blake N. Johnson ${ }^{\text {a,d }}$, \\ Timothy E. Long ${ }^{\mathrm{a}, \mathrm{b}}$, Christopher B. Williams ${ }^{\mathrm{a}, \mathrm{e}, *}$ \\ a Macromolecules Innovation Institute at Virginia Tech, 1075 Life Sciences Cir, Blacksburg, VA 24061, United States of America \\ b Department of Chemistry, Virginia Tech, 1040 Drillfield Dr., Blacksburg, VA 24061, United States of America \\ c Department of Chemical Engineering, Virginia Tech, 635 Prices Fork Rd., Blacksburg, VA 24061, United States of America \\ d Grado Department of Industrial and Systems Engineering, Virginia Tech, 1145 Perry St., Blacksburg, VA 24061, United States of America \\ e Department of Mechanical Engineering, Virginia Tech, 635 Prices Fork Rd., Blacksburg, VA 24061, United States of America
}

\section{H I G H L I G H T S}

- Combines IR thermography and heattransfer modeling to estimate temperature profiles from ten laser parameter combinations.

- Predicts the extent of coalescence at the onset of physical gelation for nylon-12 from the ten temperature profiles.

- Introduces the "critical coalescence ratio" to link process settings with final part properties via predicted coalescence.

- The method is demonstrated using nylon-12 as an example polymer, but is polymer agnostic.

\section{A R T I C L E I N F O}

\section{Article history:}

Received 25 September 2020

Received in revised form 1 December 2020

Accepted 10 January 2021

Available online 21 January 2021

\section{Keywords:}

Additive manufacturing

Powder bed fusion

Selective laser sintering

Polymer coalescence

Process parameter prediction

Physical gelation
GRAPHICALABSTRACT

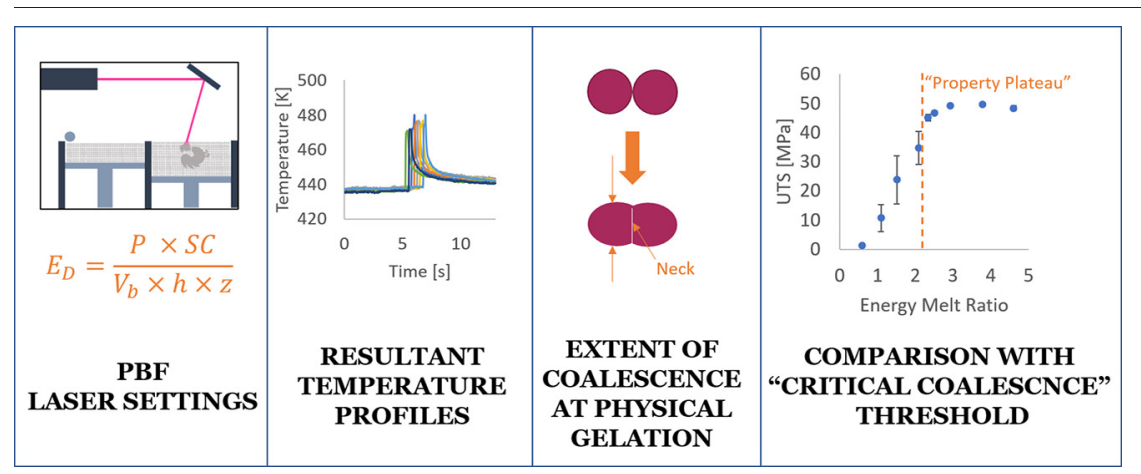

\begin{abstract}
A B S T R A C T
The state of the art in property-process relationships in the laser polymer powder bed fusion (LPPBF) subcategory of powder bed fusion (PBF) has derived relationships between the energy supplied and polymer thermal properties governing melting and degradation, so-called the "energy melt ratio (EMR)." The EMR provides a framework for process parameter value selection based solely on melting behavior. However, coalescence, and not merely melting, is the basis for mechanical properties in LPPBF printed parts. The authors present a method for (1) predicting polymer coalescence based on transient temperature profiles resulting from a combination of LPPBF process parameter values and (2) connecting the predicted coalescence response to the observed onset of a plateau in mechanical properties. This work tests the hypothesis that the observed onset of a mechanical property plateau corresponds with a transition in consolidation physics. Complete coalescence must be achieved prior to the onset of physical gelation. For this work, in situ transient temperature profiles were obtained using infrared thermography. Coalescence prediction, via the Upper-convected Maxwell model, and physical gelation prediction, via Lauritzen-Hoffman and Avrami equations, were found to successfully identify LPPBF parameter combinations resulting in parts with density and tensile strength inside the plateau region. The hypothesis that the plateau occurs at the onset of closed pore morphology is supported.
\end{abstract}

(c) 2021 Published by Elsevier Ltd. This is an open access article under the CC BY-NC-ND license (http:// creativecommons.org/licenses/by-nc-nd/4.0/).

\footnotetext{
* Corresponding author at: Macromolecules Innovation Institute at Virginia Tech, 1075 Life Sciences Cir, Blacksburg, VA 24061, United States of America.

E-mail address: cbwill@vt.edu (C.B. Williams).
} 


\section{List of Abbreviations}

\begin{tabular}{|c|c|}
\hline $\mathrm{a}$ & Particle size \\
\hline$a_{0}$ & original particle size \\
\hline AM & Additive manufacturing \\
\hline CCR & Critical Coalescence Ratio \\
\hline De & Deborah number \\
\hline DSC & Differential scanning calorimetry \\
\hline$E_{D}$ & Energy density \\
\hline EMR & Energy melt ratio \\
\hline h & Hatch spacing \\
\hline IR & infrared \\
\hline 1 & layer height \\
\hline $\mathrm{P}$ & laser power \\
\hline PBF & Powder bed fusion \\
\hline LPPBF & Laser polymer powder bed fusion \\
\hline$r_{\text {chamber }}$ & $\begin{array}{l}\text { Dimensionless neck radius after isothermal time as a supercooled } \\
\text { polymer melt }\end{array}$ \\
\hline$r_{\text {critical }}$ & Dimensionless neck radius at 0.94 relative density \\
\hline$r_{\text {laser }}$ & Dimensionless neck radius after laser scanning \\
\hline SC & Scan count \\
\hline$t_{A F, 1}$ & Time available for fusion while the laser is scanning \\
\hline$t_{A F, 2}$ & $\begin{array}{l}\text { Time available for fusion after the laser has finished scanning until a new } \\
\text { layer begins }\end{array}$ \\
\hline$t_{A F}$ & Time available for fusion \\
\hline$T_{b, \text { inf }}$ & Temperature of the powder bed bulk \\
\hline$t_{C F}$ & Time for critical fusion (i.e., 0.835 dimensionless neck radius) \\
\hline$T_{\text {feed }}$ & Temperature of the feed powder \\
\hline$t_{X 10}$ & Time to $10 \%$ crystallinity \\
\hline$T_{m}^{0}$ & Equilibrium melting temperature \\
\hline$T_{b}$ & Surface temperature of the powder bed \\
\hline$T_{\max }$ & maximum temperature measured when laser scanning \\
\hline UCM & Upper-convected Maxwell \\
\hline$V_{b}$ & Beam speed \\
\hline $\mathrm{x}$ & neck radius \\
\hline$\eta$ & Viscosity (unspecified) \\
\hline$\eta_{0}$ & Zero-shear viscosity \\
\hline$\eta_{\text {ext }}$ & Extentional viscosity \\
\hline$\eta_{s s}$ & Steady shear viscosity \\
\hline$\Gamma$ & Surface energy \\
\hline$\lambda$ & Relaxation time \\
\hline$\Theta$ & Angle between coalescing particles \\
\hline
\end{tabular}

\section{Introduction}

Powder bed fusion (PBF) additive manufacturing (AM) is one of seven technology families classified by ASTM/ISO 52900 [1]. PBF repeats the sub-functions of (1) powder recoating, (2) energy input/absorption, and (3) particle consolidation to fabricate objects on a layer-by-layer basis. Consolidation, or "fusion", must occur in both neighboring particles within one layer and across layers, to form mechanically robust 3D structures. This manuscript focuses on the most prevalent polymer
PBF sub-category, laser polymer powder bed fusion (LPPBF) (a.k.a. polymer laser sintering), in which consolidation occurs via particles melting and flowing together through a selective increase in molecular motion caused by a scanning infrared (IR) laser beam (conceptually depicted in Fig. 1). The work presented in this manuscript describes a method for predicting the extent of consolidation from experimentally measured temperature profiles resulting from laser scanning during LPPBF manufacturing. Additionally, the predicted extent of consolidation is connected to mechanical performance of printed parts via the threshold that delimits two modes of consolidation: viscous coalescence and bubble diffusion.

Consolidation in LPPBF governs final part density, and thereby mechanical and other performance properties. Partial particle fusion results in an inhomogeneous structure full of defects. The effect of partial melting (and its influence on mechanical properties) for LPPBF nylon-12 has been reported in literature by Zarringhalam and coauthors $[2,3]$. Additional research has attributed poor mechanical properties to insufficient particle coalescence $[4,5]$.

Fig. 2a-b depicts aggregated ultimate and yield tensile strength as a function of surface energy density reported from published literature of LPPBF-processed nylon-12. The trend among all published data cited in Fig. 2a-b matches the description given by Starr, et al.: an initial steep slope followed by a region where tensile properties are generally independent of energy density [6]. Although Starr, et al. observed the trend, they did not hypothesize a rationale for its occurrence. Since mechanical and other performance properties are governed by density, the authors hypothesize the change in scaling behavior between the mechanical properties and "manufacturing energy" shown in Fig. 2a-b is a reflection of a change in the consolidation behavior of polymer particles.

Verbelen and coauthors used their developed, dimensionless normalization factor, the energy melt ratio (EMR), to correlate and explain the process-property relationship between LPPBF laser parameters and ultimate tensile properties. The EMR is the ratio of laser energy to the energy required to melt a defined control volume of polymer powder at a predetermined chamber temperature. Theoretically, printing parts using an EMR at or above one will result in full particle melting; however, Vasquez, et al. reported maximum tensile properties at EMR close to 4.0 [7]. This implies that, although an EMR of 1.0 results in molten particles, additional energy is required for full coalescence and maximum properties. By example, Chatham and coauthors use the EMR to predict the range of acceptable energy densities for printing poly (phenylene sulfide) [13]. However, they note that the minimum energy for melting $(E M R=1)$ did not produce a homogeneously coalesced layer of particles and suggest the low prediction may be improved through incorporating rheological properties into the process parameter value selection process.
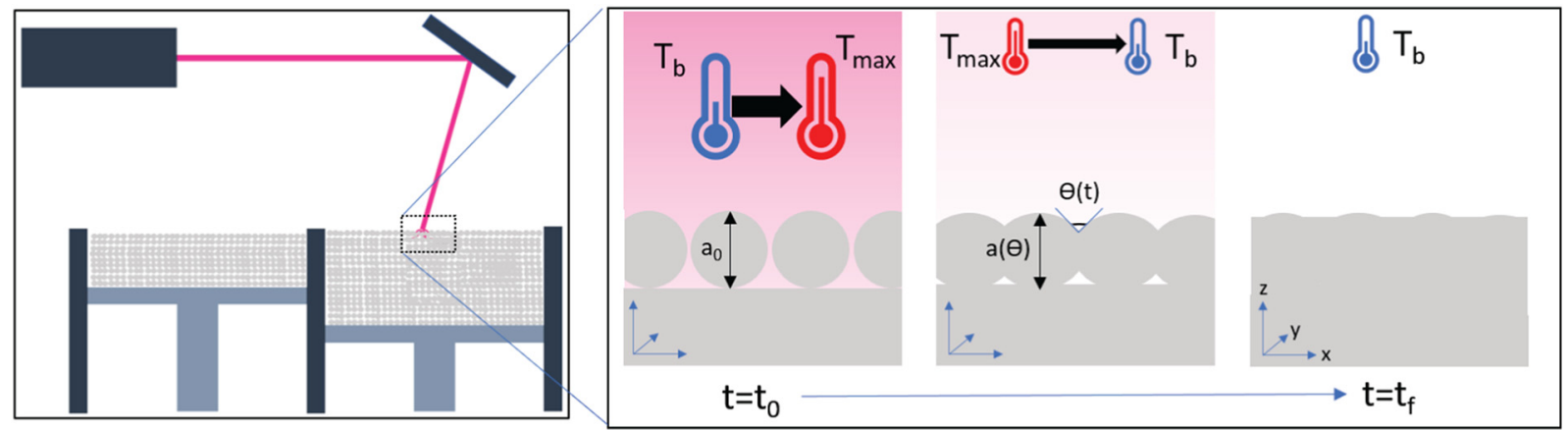

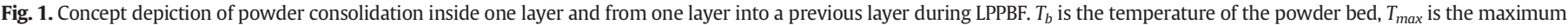

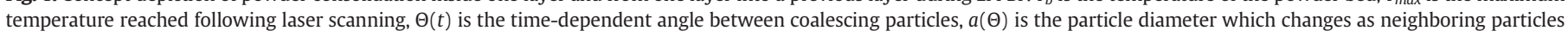
coalesce from its initial diameter, $a_{0}$. The coalescence process takes place over some timespan $t_{0}$ to $t_{f}$. 
(a)

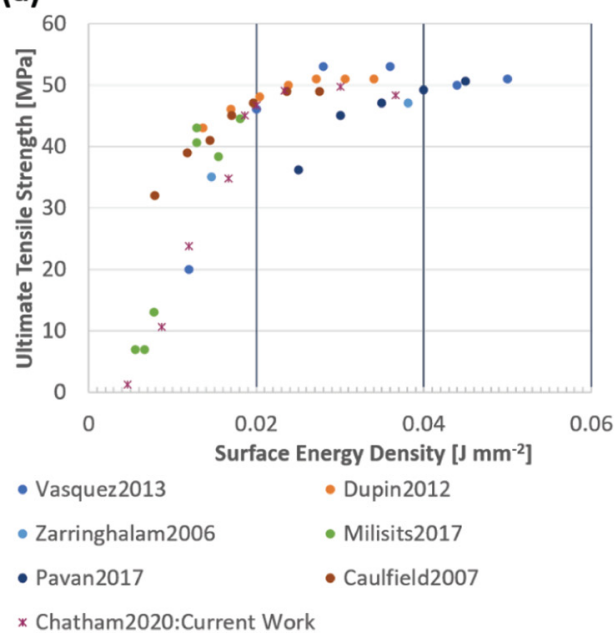

(b)

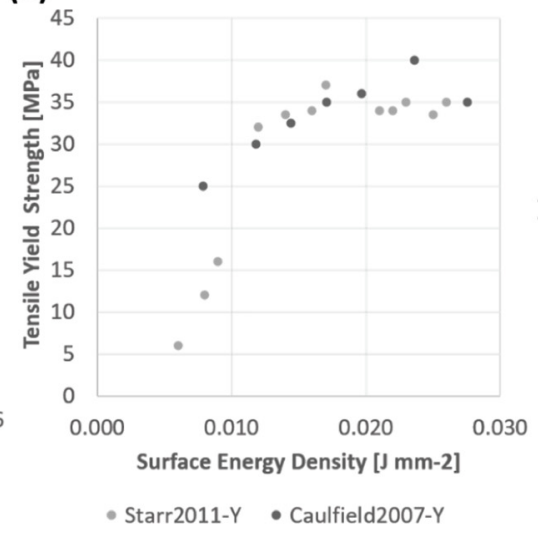

(c)

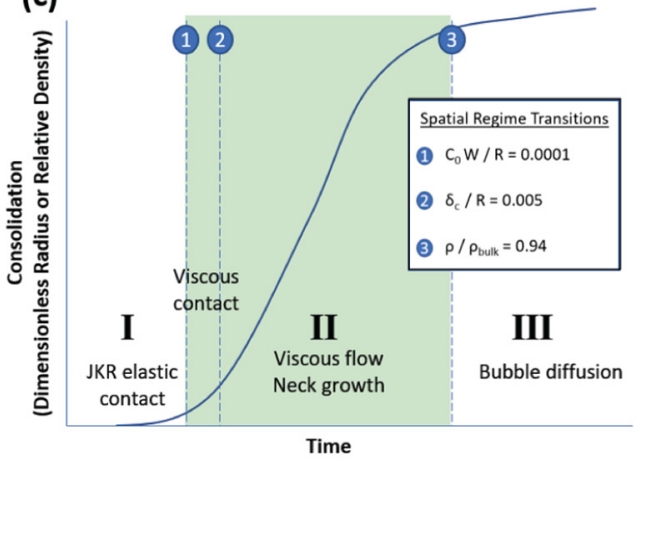

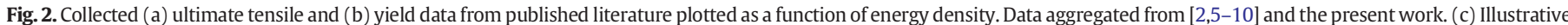

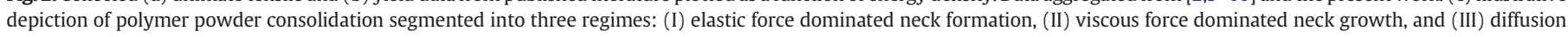

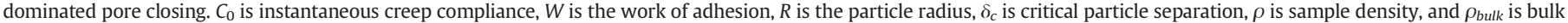
polymer density. Concepts and equations compiled from papers written by Lin, et al. [11] and Scherer [12].

Polymer consolidation takes a sigmoidal form divided into the three regimes, as depicted in Fig. 2c, with viscous neck growth (Regime II) being the overall dominating regime. The articles by Lin and coauthors [11] and Kamyabi and coauthors [14] thoroughly review and describe the first two stages of isothermal polymer particle consolidation. Final stage densification described by bubble diffusion physics has been reported on by Kontopoulou, et al. [15] and Ly, et al. [16,17]. Viscous effects dominate consolidation after the product of instantaneous creep compliance and work of adhesion equals one-tenthousandth of the particle radius [11] (Transition 1 in Fig. 2c) and remain dominate until closed pores trap air around 0.94 relative density [12] (Transition 3 in Fig. 2c). The work presented in this manuscript will focus on the effects of LPPBF-characteristic manufacturing stimuli on viscous particle coalescence, as this regime has the largest influence on mechanical properties of printed parts. The authors present the hypothesis that the observed change in dependence of mechanical properties on laser energy density described in prior work $[5,7,18,19]$ (Fig. 2a-b) coincides with the transition from viscous coalescence to bubble diffusion (Transition 3 in Fig. 2c). The authors also hypothesize that this transition can be predicted from intrinsic polymer properties that govern viscous coalescence in relation to any given set of process parameter values.

\subsection{Viscous coalescence}

LPPBF affects an ambient pressure, inert atmosphere (e.g., constant nitrogen purge) environment for particle consolidation. As such, LPPBF is similar to rotational molding, an older, traditional manufacturing process based on low-pressure coalescence of polymer powder at elevated temperature [20]. Due to these similarities, models originally developed for rotational molding may be adapted into a LPPBF context. Physical models describing consolidation in the context of rotational molding are often based on the Frenkel model for viscous sintering [21]. This model describes coalescence of two identical, spherical particles driven by surface energy and opposed by viscosity. The Frenkel model has been improved and adapted for many different contexts since it was developed in 1945. The history of coalescence modeling is described in detail by Kamyabi and coauthors [14]. For rotational molding, the adaptions of the Frenkel model to include viscoelastic effects by Bellehumer [22] and Scribben [23] are recent and well-cited. These models have served as a foundation for modeling two-particle coalescence in LPPBF; however, LPPBF differs from rotational molding in the key areas of (1) on average, smaller powder size (e.g., $\sim 45-90 \mu \mathrm{m}$ vs $\sim 300-500 \mu \mathrm{m}$ ), (2) highly transient temperature profile, and (3) layerwise paradigm. To achieve maximum performance from LPPBF printed parts, full particle consolidation must be achieved through appropriate selection of the build parameters.

The polymer properties governing viscous coalescence behavior are represented in the Deborah number $(D e)$ for coalescence shown in Eq. (1), where $\lambda$ is the characteristic relaxation time, $\Gamma$ is surface energy, $\eta_{0}$ is zero-shear rate viscosity, and $a_{0}$ is the initial particle diameter.

$D e=\frac{\lambda \Gamma}{\eta_{0} a_{0}}$

The key to understanding coalescence in the LPPBF context is understanding the response of these four properties to the unique physical aspects of LPPBF manufacturing. LPPBF's characteristic particle size of 45-90 $\mu \mathrm{m}$ average diameter [24], which is smaller than most rotational molding powders, favors coalescence. Particle size is perhaps the easiest property to investigate, as it remains constant across a wide temperature range; however, values for the other three properties vary widely depending on temperature. This makes modeling them in a LPPBF context challenging due to highly transient laser heating and subsequent cooling.

\subsection{Prior work in $L P P B F$ coalescence simulation}

The Upper-convected Maxwell (UCM) equations have been used to model coalescence in both rotational molding $[23,25,26]$ and LPPBF [27]. The UCM model mathematically represents the behavior of a viscoelastic material under large deformations (e.g., diffusive motion), such as found in two-particle coalescence. Although the UCM was intended to model shear flow, it has been reported in literature to also describe biaxial extentional flow found in rotational molding [26] and LPPBF [27]. In general, solving the UCM for any particular load case reveals the polymer's response is dictated by the four constituent properties of the Deborah number: surface energy, relaxation time, viscosity, and some characteristic length. Powder coalescence has been characteristically identified as biaxial extension [23], therefore polymer properties representative of the biaxial extention load case should be used to understand and predict the extent of consolidation in LPPBF. This means using reptation time $(\lambda)$ (or longest relaxation time from steady- 
shear) as the characteristic relaxation time of diffusive movement and extensional viscosity at low rates $(\eta)$. Although extensional viscosity is often difficult to experimentally measure, the Trouton ratio (i.e., $\eta_{\text {ext }}=6 \times \eta_{s s}$ ) may be used to approximate bi-axial extensional viscosity from steady-shear viscosity at low shear rates [23]. The Trouton ratio approximation of biaxial viscosity is most accurate at low shear rates where the polymer melt behaves in a Newtonian manner. The approximation can deviate from true biaxial viscosity if the polymer is prone to extentional strain hardening; however, significant strain hardening of polymer melts is generally uncommon at the shear rates present in LPPBF [28].

Prior work in modeling coalescence during LPPBF typically uses the simplified case of two-particle coalescence to avoid the computationally expensive calculations of simultaneous, multi-particle interactions. In the two-particle coalescence models by Bellehumeur [22] and Scribben [23], the characteristic length is the particle diameter, and the overall material deformation is quantified via the angle between two adjacent particles, and its rate of change (see Fig. 1). In the Bellehumeur and Scribben models, the angle between adjacent particles is trigonometrically converted into the radius of contact area between particles, also termed the "neck." Neck growth rate, normalized to some other characteristic length of the two particle system, is the method most frequently used for describing coalescence rate.

Alternatively, densification behavior can be evaluated via diminishing porosity, as done by Mokrane, et al. in their numerical simulation of LPPBF for nylon-12 [27]. They build upon work by Scherer and coauthors using the UCM equation in their porosity evolution model [12]. Tontowi and Childs, and Sun and coauthors also take after Scherer's work with their volumetric conservation of a body centered cubic packed powder bed $[29,30]$. Sun, et al.'s model introduces a new variable that accounts for partial fusion and adjusts the particle contact surface area. Printed part density versus energy density curves simulated by Tontowi and Childs at three different bed temperatures highlight the importance of bed temperature in achieving fully dense parts [29].

Work presented here connects transient temperature profiles generated from a variety of LPPBF machine parameter combinations to the UCM two-particle coalescence model. The UCM model was selected because of its extensive use in other forms of polymer powder processing and its incorporation of polymer relaxation time, which is missing from Sun's partial sintering model. Prior work has mainly informed models from material measurements $[8,26,29]$, or has characterized transient temperature profiles resulting from different processing conditions [31,32], but only the multi-physics modeling work of Mokrane and coauthors [27] informed models from both material and machine parameter measurements. Multi-physics models are critical for accurately representing the complexities of semi-crystalline polymers typically used in LPPBF processing, as crystallization significantly opposes coalescence. This is discussed in greater detail in Section 1.3.

LPPBF's smaller average powder size compared to rotational molding should increase the accuracy of the UCM model for LPPBF application over rotational molding, as the smaller particle size increases the validity to assume lumped capacitance. The UCM assumes isothermal coalescence and does not account for additive layering; therefore, the temperature profile resulting from LPPBF processing will be analyzed in isothermal segments to predict coalescence using the UCM.

\subsection{Crystallization kinetics arresting diffusive molecular motion}

Coalescence requires diffusive molecular motion and is retarded or arrested by anything restricting molecular mobility. This is generally discussed in terms of viscosity, especially for amorphous polymers. However, when crystallization occurs in crystallizable polymers, crystallites act as physical crosslinks that severely restrict molecular motion. Prior work studying melt spinning of crystallizable thermoplastic polymers has noted that volume fractions between 0.2 and $10 \%$ can completely arrest polymer flow [33,34]. This value can be experimentally determined as "physical gelation time." This value is also estimated in process modeling literature to be $10 \% \mathrm{v} / \mathrm{v}$ based on empirical observation $[34,35]$.

The influence of crystallization arresting coalescence in LPPBF has been noted in the multi-physics LPPBF process models published by Mokrane and coauthors [27]. Their work describes LPPBF as a quasiisothermal process with volumetric heat generation due to laser scanning, which melts polymer particles resulting in viscous flow behavior modeled by the UCM equations and opposed by Lauritzen-Hoffman crystallization [27]. In the present work, the authors will take the ideas represented in Mokrane, et al.'s model one step further, using aspects of each constituent model representation of the LPPBF process to connect the process parameter combination affected temperature to printed part properties.

\subsection{Roadmap}

All forms of prior work investigating polymer coalescence in the LPPBF context have helped to provide a framework for understanding both the LPPBF stimuli imposed on polymer powder and the polymer's response during manufacturing. However, a quantitative framework for predicting the extent of consolidation and resultant performance properties from specific combinations of process parameter values has not yet been developed. While the energy melt ratio (EMR) has provided a framework to choose LPPBF process parameter values in the context of melting and thermal degradation with the goal of repeatably predicting the performance properties of printed parts, it does not consider polymer rheological properties in its calculations. This manuscript describes the first steps toward informing process parameter value selection from two-particle coalescence behavior. The proposed framework is based on the hypothesis that the transition in consolidation regime from viscous coalescence to bubble diffusion coincides with the observed onset of "mechanical property plateau" (see Fig. 2). This framework connects the temperature-dependent properties of LPPBF polymer feedstock with thermal manufacturing stimuli to predict the performance properties of printed parts. This will be presented as the ratio between predicted dimensionless neck radius at physical gelation to the dimensionless neck radius approximating the transition from "viscous coalescence" to "bubble diffusion" consolidation physics and will be termed "critical coalescence ratio" (CCR). Using this definition, the authors hypothesize that all process parameter value combinations achieving a CCR at or above 1 will result in mechanical properties inside the "mechanical property plateau," close to maximum achievable properties. The CCR relates predicted dimensionless neck radius from a measured transient temperature profile to Scherer's threshold for closed pore morphology (Transition 3 in Fig. 2c).

The authors use in situ IR thermography to characterize the imposed temperature profile from a variety of laser parameter combinations on polymer powder. These profiles are used to determine two-particle coalescence and crystallization rates, which are in turn used to assess the timescale of bi-axial coalescence in the context of LPPBF's timescale of manufacturing. A detailed discussion of using in situ IR thermography combined with heat transfer modeling to obtain the transient temperature profiles is provided elsewhere [36]; this document focuses on using the measured and modeled temperature data to predict tensile properties resulting from several laser parameter value combinations. Mechanical test specimens are fabricated from the same laser parameter combinations used to generate the input temperature profiles. Trends in printed density, ultimate tensile strength, elongation at break, and modulus are interpreted based on the CCR.

\section{Proposed method to identify laser parameters for critical coalescence}

The proposed "Critical Coalescence Ratio" (CCR) (Eq. (2)) connects the machine stimuli and material response of polymers by comparing 
the calculated dimensionless neck radius affected by a given transient temperature profile to the dimensionless neck radius threshold value for onset of closed pores according to Scherer's model $\left(r_{\text {critical }}\right)$ [12].

$$
\begin{aligned}
& C C R=\frac{1}{r_{\text {critical }}} \times \sum_{1}^{n}\left(r_{\text {laser }}+r_{\text {chamber }}\right) \\
& \text { while } \sum_{i=1}^{n} t_{i}<t_{A F}
\end{aligned}
$$

A CCR at or above 1 indicates that a set of print parameters will coalesce the polymer powder into the bubble diffusion regime and exhibit mechanical properties in the plateau region. The ratio is comprised of two parts corresponding to the two methods of energy input in LPPBF (i.e., laser scanning and elevated chamber temperature), pertaining to the two regimes depicted in Fig. 4. The resultant dimensionless neck radius $\left(r_{\text {laser }}+r_{\text {chamber }}\right)$ is calculated at the relevant transient temperature profile until diffusive molecular motion is arrested by the physical gelation. The time from initial laser scanning to physical gelation is termed "time available for fusion" $\left(t_{A F}\right)$. The competing phenomena of coalescence and crystallization are calculated over a series of $n$ isothermal events (see Fig. 4). This calculation is performed for every layer completed within the span of $t_{A F}$, where $n$ is the number of isothermal segments between $t=0$ and $t_{A F}$.

The guiding hypotheses for this work are presented in Fig. 3. This figure summarizes the evaluation methods in part as an experimental flowchart. Various aspects of proposed experimental and model work have been previously reported in literature for the LPPBF context; however, the primary novelty of the current work is connecting all these aspects together to explain the commonly observed change in scaling behavior.

The idealized transient temperature profile for a single layer of the quasi-isothermal LPPBF process is presented in Fig. 4. Note the division between (I) laser-induced heating and (II) supercooled meltpool. This division represents the "quasi-isothermal" nature of LPPBF as a series of temperature spikes. So long as the temperature increases above equilibrium melting temperature $\left(T_{m}^{0}\right)$, particle fusion will continue after laser scanning as a supercooled polymer melt. The overall $t_{A F}$ will be the summation of both laser scanning and decay $\left(t_{A F, 1}\right)$ and isothermal supercooled melt $\left(t_{A F, 2}\right)$. Ideally, this should also include the time to achieve full melting of polymer powder, but this is assumed to be negligible in the present work as the time to reach peak temperature after laser scanning is less than $0.5 \mathrm{~s}$.

Critical fusion occurs when powders are coalesced to 0.94 relative density. This value is based on Scherer's work [12]. Each dimensionless neck radius component in Eq. (2) can be re-written as:

$r_{\text {laser }}=\int_{0}^{t_{A F, 1}}\left(\frac{x}{a}\right)^{\prime}(T(t)) \cdot d t$

$r_{\text {chamber }}=\int_{t_{A F, 1}}^{t_{A F, 2}}\left(\frac{x}{a}\right)^{\prime}(T(t)) \cdot d t$

$r_{\text {critical }}=\left(\frac{x}{a}\right)_{\text {critical }}$

where $x$ is the radius of the contact area between particles (aka, "neck"), $a_{0}$ is the initial particle radius, $a$ is the time-dependent particle radius, and $\left(\frac{x}{a}\right)$ ' is rate of change of dimensionless neck radius, which is determined over a variety of temperatures via the UCM equations. This is discussed in greater detail in Section 4.2 with the UCM results presented in Fig. 6a and calculated $\left(\frac{x}{a}\right)^{\prime}(T)$ in Fig. 6b. The details of the UCM are included as Supplemental Information. Both energy input components will be discussed in greater detail in their own specific sections (Sections 2.1 and 2.2). Critical dimensionless neck radius affecting 0.94 relative density is calculated to be 0.835 as detailed in supplemental information. Fig. 4 depicts graphically what Eqs. (3)-(5) describe mathematically.

\subsection{Laser-induced heating}

The first energy input aspect of LPPBF considered is laser-induced heating. Infrared thermography is used in this work to quantify the transient temperature profile of the powder bed surface resulting

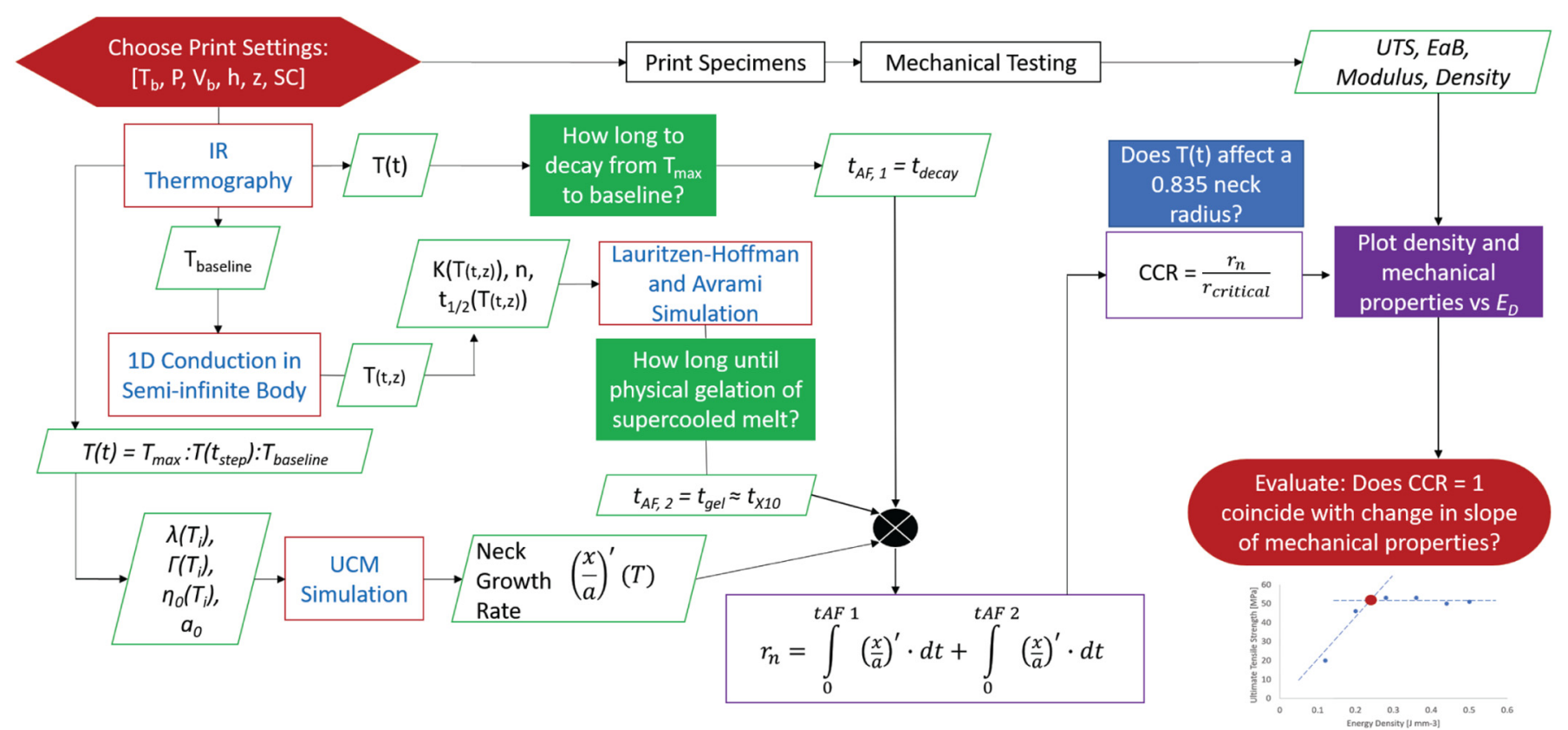

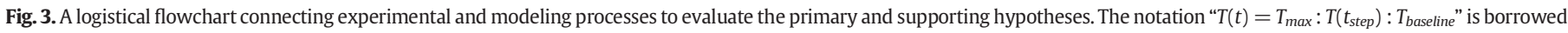

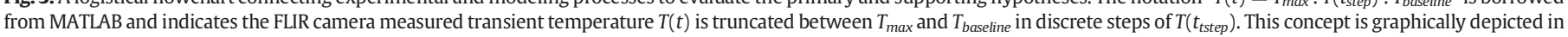
Fig. 4. 


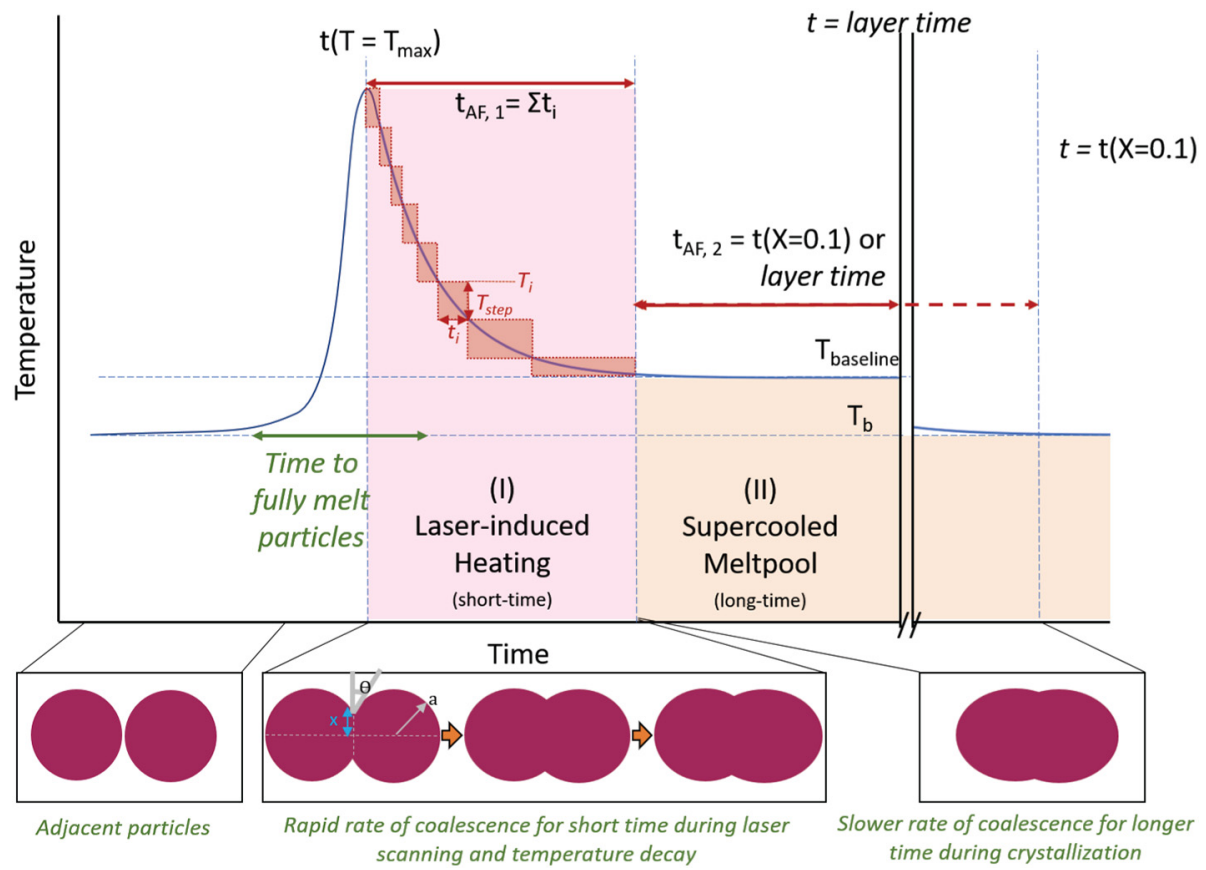

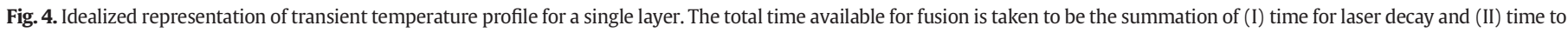
reach $10 \%$ crystallinity (i.e., physical gelation) at isothermal baseline temperature $(t(X=0.1))$. Time to $10 \%$ crystallinity will likely occur over multiple layers.

from a variety of print parameter settings. As IR thermography only measures surface temperature, the complete transient temperature profile is estimated via a combination of IR thermography and thermal modeling by approximating the LPPBF process as conduction into a semi-infinite body. The detailed description of generating the transient temperature profiles used in this report are included in supplemental information and [36]. For this study, variations in energy density are accomplished by only changing laser power. As depicted in the flowchart (Fig. 3), each temperature within the transient temperature profile $\left(T_{i}\right)$ is used to inform modeling and experimental methods used to obtain dimensionless neck growth rate $\left(\left(\frac{x}{a}\right)^{\prime}\right)$ and $t_{A F}$.

To calculate the temperature-dependent neck growth rate, the decay from $T_{\max }$ to $T_{\text {baseline }}$ is divided into discrete steps in time and temperature (Fig. 4). Isothermal dimensionless neck growth rates can be determined through UCM simulation at temperatures spanning $T_{\max }$ to $T_{\text {baseline }}$; this is represented as $\left(\frac{x}{a}\right)^{\prime}(T)$ in Eq. (6). Each $\left(\frac{x}{a}\right)^{\prime}(T)$ comprises a fraction of the total neck growth rate according to the amount of time spent at temperature $T=T_{i}$; therefore, the integral in Eq. (2) is approximated by Eq. (6), where the sum of all $\Delta t_{i}$ is equal to the decay time and $n$ is the total number of isothermal segments.

$r_{\text {laser }}=\int_{0}^{t_{A F, 1}}\left(\frac{x}{a}\right)^{\prime}(T(t)) \cdot d t \approx \sum_{i=1}^{n}\left(\frac{x}{a}\right)^{\prime}\left(T=T_{i}\right) \cdot \Delta t_{i}$

Due to additive layering, brief spikes in temperature are imposed on any given control volume of polymer over several layers, depending on the penetration depth of the laser beam into the powder bed, the thermal penetration depth dictated by thermal diffusivity, and layer time of the build-specific toolpath. The combined effect of these short-term increases in temperature is important to consider to evaluate total time available for fusion.

\subsection{Fusion of the supercooled melt}

The second aspect of LPPBF is continued fusion in the supercooled melt between laser scanning events. Statements have been made in prior LPPBF literature hypothesizing that the laser scanned polymer remains in the melt state for the entire build. Such statements are not supported by polymer crystallization kinetics and have been refuted in published literature $[37,38]$. Therefore, understanding the interconnected roles of crystallization and fusion in LPPBF will be investigated in this study.

The short time of LPPBF laser-induced heating results in a supercooled polymer melt pool in the shape scanned by the laser. Although the temperature of the scanned area is close to the temperature of the surrounding powder, the thermal history of the scanned area creates an actively coalescing and simultaneously crystallizing supercooled melt pool. Although set by the machine operator as $T_{b}$ (the bed's surface temperature) and $T_{b \text {, inf }}$ (the temperature in the bulk of the powder bed), and generally invariant over the course of a build, LPPBF's characteristic additive layering results in a local increase in temperature resulting from repeatedly scanning the same cross-section. The magnitude of temperature increase is dependent on the laser scan settings and toolpath. The authors refer to the local temperature of the supercooled melt as $T_{\text {baseline }}$ to distinguish it from the machine set temperatures $T_{b}$ and $T_{b}$, inf. $T_{\text {baseline }}$ is estimated from the combined IR and heat transfer modeling summarized in supplemental information.

In this regime, temperature slowly cools from $T_{\text {baseline }}$ to $T_{b \text {, inf. }}$ This is a smaller change in temperature than observed during laser scanning and it occurs over a far longer time; therefore coalescence is slowed and ultimately arrested by crystallization. The amount of crystallization required to arrest diffusive molecular motion can be thought of as "physical gelation" [33,34]. This is estimated to be between 2 and $10 \%$ $v / v$ for most polymers $[33,34,39]$. The authors chose to use "time to $10 \%$ relative crystallinity $\left(t_{X 10}\right)$ " to predict the physical gelation time of nylon-12 based on prior work for other nylons by Hirayama and coauthors [39]. Hirayama reports critical volume fraction of crystalline domains for gelation for nylon- 6 to be around $5 \% \mathrm{v} / \mathrm{v}$ for a $50 \mathrm{~kg} \mathrm{~mol}^{-1}$ polymer [39] and around $15 \% \mathrm{v} / \mathrm{v}$ for $100 \mathrm{~kg} \mathrm{~mol}^{-1}$, which represents the range of molecular weights reported for LPPBF-grade nylon-12 ( 19-65 kg mol-1 [18], 70-90 kg mol ${ }^{-1}$ [2], 75-129 $\mathrm{kg} \mathrm{mol}^{-1}$ [40]); therefore, the authors judge $10 \%$ as a reasonable approximation for nylon- 12 . As $10 \% \mathrm{v} / \mathrm{v}$ is greater than $10 \%$ relative crystallinity, this will be a conservative estimate. Time to reach $10 \%$ relative crystallinity 
will be used as the ultimate limiter for $t_{A F}$, and will likely require several layers to reach. Therefore $t_{A F, 2}$ in Eq. (2) will be (layer time $-t_{A F, 1}$ ) for each layer until this value exceeds $t_{X 10}$.

\section{Experimental methods}

The aim of experimentation in this work is to determine if the density and mechanical properties of parts produced using LPPBF print parameter value combinations resulting in a CCR at or above 1 will fall in the "mechanical property plateau" region when results are plotted against EMR or energy density (See Fig. 2). Therefore, both the predicted dimensionless neck radius at physical gelation and the mechanical properties of printed parts must be determined for a variety of LPPBF process parameter value combinations. The overarching outline of experimentation to predict dimensionless neck radius from LPPBF process parameter value combination proceeds through the steps outlined in Fig. 3. Density and mechanical properties will be conventionally evaluated as described in Sections 3.7 and 3.8.

\subsection{Materials}

A commercial "powder bed fusion grade" polyamide-12 (PA12) (a.k. a. nylon-12) was obtained from Advanced Laser Materials (ALM) under the trade name PA 250. Thermal properties were characterized using a TA Instruments Q 2000 differential scanning calorimeter (DSC) according to a typical heat-cool-heat experiment at $10 \mathrm{~K} \mathrm{~min}^{-1}$ from 273 to $523 \mathrm{~K}\left(0-250{ }^{\circ} \mathrm{C}\right)$. A DSC curve of the as-received powder is included in supplemental information. Typical glass transition temperature for nylon-12 is around $323 \mathrm{~K}\left(50^{\circ} \mathrm{C}\right)$. Onset and peak melting temperatures are observed to be 447 and $454 \mathrm{~K}\left(174,181^{\circ} \mathrm{C}\right)$, respectively. The specific heat capacity signal from the cooling step was exported from TA Universal Analysis software and used as a look-up table for temperature-dependent specific heat capacity when modeling heat transfer.

In addition, fractional melting behavior was characterized using the "running integral" analysis option in TA Instruments Universal Analysis (see supplemental information for plot). The area percent of the melting peak up to the measured $T_{\max }$ for a given laser parameter combination was assumed to be fractional melting. This value is important in the discussed of crystallization in Section 4.4.

\subsection{Powder characterization}

Particle size distribution was measured for as-received powder using a Horiba LA-950 static laser light particle size analyzer. Distributions reported are constructed from volume-based calculations with water as the fluid media. Refractive index of nylon-12 was taken to be 1.5 based on literature precedent $[41,42]$. The particle size at $10 \%, 50 \%$ (a.k.a. average particle size), and $90 \%$ of the whole sample by volume are shown in Table 1. Alongside this information are the apparent and tapped densities of the nylon-12 powder. These were determined according to ASTM D1895 [43] and an adapted version of S.3.6 World Health Organization Pharmacopoeia method [44], respectively.

Table 1

Powder and thermal properties of PA 250, a commercially available LPPBF grade nylon-12 powder. These properties are used to calculate EMR.

\begin{tabular}{llll}
\hline Powder property & Value & Thermal property & Value \\
\hline$d_{10}[\mu \mathrm{m}]$ & 35 & Enthalpy of Fusion $[\mathrm{J} / \mathrm{g}]$ & 98.05 \\
$d_{50}[\mu \mathrm{m}]$ & 62 & Specific Heat $\left[\mathrm{J} \mathrm{g}^{-1}{ }^{\circ} \mathrm{C}^{-1}\right]$ & 2.06 \\
$d_{90}[\mu \mathrm{m}]$ & 317 & End of Melt Peak $\left[{ }^{\circ} \mathrm{C}\right]$ & 190 \\
Apparent Density $\left[\mathrm{g} \mathrm{cm}^{-3}\right]$ & 0.487 & & \\
Tapped Density $\left[\mathrm{g} \mathrm{cm}^{-3}\right]$ & 0.594 & & \\
\hline
\end{tabular}

Table 2

Chosen print settings and resulting energy densities and energy melt ratios.

\begin{tabular}{|c|c|c|c|c|c|c|}
\hline \multicolumn{7}{|c|}{ Constant parameters } \\
\hline$T_{\text {feed }}$ & $T_{b, \text { inf }}$ & $T_{b}$ & $V_{b}$ & $\mathrm{~h}$ & 1 & $\mathrm{SC}$ \\
\hline $\begin{array}{l}\mathrm{K} \\
\left(155^{\circ} \mathrm{C}\right)\end{array}$ & $\begin{array}{l}433 \mathrm{~K} \\
\left(162^{\circ} \mathrm{C}\right)\end{array}$ & $\begin{array}{l}443 \mathrm{~K} \\
\left(170^{\circ} \mathrm{C}\right)\end{array}$ & $5 \mathrm{~m} \mathrm{~s}^{-1}$ & $0.300 \mathrm{~mm}$ & $0.102 \mathrm{~mm}$ & 1 \\
\hline $\mathrm{P}[\mathrm{W}]$ & & & $E_{D}\left[\mathrm{~J} \mathrm{~mm}^{-2}\right]$ & & & EMR \\
\hline 3 & & & 0.00200 & & & 0.25 \\
\hline 7 & & & 0.00467 & & & 0.59 \\
\hline 13 & & & 0.00867 & & & 1.09 \\
\hline 18 & & & 0.0120 & & & 1.51 \\
\hline 25 & & & 0.0167 & & & 2.09 \\
\hline 28 & & & 0.0187 & & & 2.34 \\
\hline 30 & & & 0.0200 & & & 2.51 \\
\hline 35 & & & 0.0233 & & & 2.93 \\
\hline 45 & & & 0.0300 & & & 3.77 \\
\hline 55 & & & 0.0367 & & & 4.60 \\
\hline
\end{tabular}

\subsection{Powder bed fusion}

Type IV dogbones and rectangular prisms were printed using a Prodways ProMaker HT2000 powder bed fusion machine according to the parameters outlined in Table 2. Parameters listed in Table 2 are empirically known to our research group to produce quality specimens. Laser power was varied to generate a range of energy densities and thereby a range of transient temperature profiles; all other print parameters were kept constant. In Table $2, T_{b}$, inf is the bulk temperature of the powder bed, $T_{b}$ is the bed surface temperature, $V_{b}$ is beam speed, $h$ is hatch spacing, $l$ is layer height, $S C$ is scan count, $P$ is laser power, $E_{D}$ is energy density, and EMR is the energy melt ratio.

Energy density and energy melt ratio values for each set of print parameters are included to contextualize and connect the present work to the work of Vasquez, et al. [7] and Starr, et al. [6]. These settings are within the predicted printable range of nylon-12 based on the upper limit determined by Vasquez, et al. (EMR $=1$ to 6.2 ) [7]. The parameter sets using $3 \mathrm{~W}$ and $7 \mathrm{~W}$ are included as negative controls; these sets of parameters should not result in mechanically stable parts, as evidenced by their $\mathrm{EMR}<1$.

\subsection{Transient temperature profiles via infrared thermography and heat transfer modeling}

Profiles of powder bed surface temperature were obtained in situ with a Micro Epsilon TIM 640 IR camera while laser scanning preheated polymer powder, in conjunction with TIM Connect software. The sampling rate of the camera is $32 \mathrm{~Hz}$. PA 250 was experimentally determined to have an emissivity of 0.955 through validation with a type- $\mathrm{T}$ thermocouple embedded in the powder bed at $442 \mathrm{~K}\left(169{ }^{\circ} \mathrm{C}\right)$. Results reported are an average of 28 replicate trials ( 7 replicates per layer; 4 layers). IR temperature measurements were used to determine boundary conditions for heat transfer equations to calculate the transient thermal gradient in the z-direction. The LPPBF process was modeled as one-dimensional conduction in the z-direction through a semi-infinite body. IR thermography was used to determine one boundary temperature and bulk powder temperature was assumed to remain constant at the set temperature. Additional information, including example plots of raw data, equations and assumptions for heat transfer modeling, and notes on MATLAB data processing, can be found in supplemental information.

\subsection{Crystallization kinetics}

In lieu of experimental data, the author leveraged published investigations of crystallization kinetics of DuraForm PA, another LPPBF-grade 
nylon-12, has been reported on by Zhao and coauthors [38]. Data published in that manuscript is used to estimate the time to reach $10 \%$ crystallinity at each baseline temperature measured from IR thermography. This was done to focus the novelty of the present work on the CCR and demonstrate the application of a "library" of material properties gathered from published sources. The equations used, their explanation, and a plain text version of the MATLAB script used to perform calculations are included in supplemental information.

\subsection{Coalescence modeling using upper-convected Maxwell equations}

The steady-state solution for the upper-convected Maxwell (UCM) equations have been outlined in papers authored by Pokluda, et al. [45]; Bellehumeur, et al. [26]; and Scribben, et al. [23]. The equation solution forms provided in those papers are included in supplemental information and used to estimate the rate of change of contact angle between two coalescing particles via fourth-order Runge-Kutta numerical approximation solved using MATLAB. Results from the simulation using a step size of 0.001 and 0.0001 at $444 \mathrm{~K}\left(171{ }^{\circ} \mathrm{C}\right)$ were compared and found to have a difference of $0.0004 \mathrm{~s}$, implying convergence. Therefore, a step size of 0.001 was used to determine neck radius at every temperature from 433 to $673 \mathrm{~K}\left(160-400{ }^{\circ} \mathrm{C}\right)$.

Similar to the authors' use of previously published data for predicting crystallization kinetics, the UCM model was seeded with input values reported in published literature for other "LPPBF-grade" nylon-12 powders. Tabulated values for each component of the Deborah number along references to their original publications are included as part of supplemental information.

\subsubsection{Surface energy [Г]}

Melt-state surface energy for LPPBF grade nylon-12 is reported by Dupin [8] in his thesis. These values were used for UCM simulation.

\subsubsection{Relaxation time $[\lambda]$}

Characteristic relaxation time for LPPBF grade nylon-12 is reported by Dupin in his thesis [8]. Dupin reports relaxation times based on cross-over modulus and Cole-Cole plots from oscillatory shear rheology in addition to the Carreau method for determining characteristic relaxation time from steady-shear rheology. Times were collected in the temperature range $443-503 \mathrm{~K}\left(170-230{ }^{\circ} \mathrm{C}\right)$. An exponential fit of time at crossover modulus versus temperature was used to generate data every $1 \mathrm{~K}$ between 433 and $673 \mathrm{~K}\left(160-400{ }^{\circ} \mathrm{C}\right)$. These data were used to seed the UCM model.

\subsubsection{Zero-shear, biaxial extensional viscosity $\left[\eta_{0}\right]$}

No reports of biaxial extensional viscosity were found for LPPBF grade nylon-12; however, Haworth and coauthors [18] and Dupin [8] report values for steady-shear viscosity at low shear rates over the temperature range $443-569 \mathrm{~K}\left(170-296{ }^{\circ} \mathrm{C}\right)$. A linear fit of $\log \left(\eta_{0}\right)$ vs temperature of the existing data was used to generate viscosity values every $1 \mathrm{~K}$ from 433 to $673 \mathrm{~K}\left(160-400^{\circ} \mathrm{C}\right)$. These values were multiplied by six (the Trouton ratio for biaxial extension) and used to seed the UCM model.

\subsubsection{Particle size $\left[a_{0}\right]$}

Initial particle size is assumed to remain constant at all temperatures relevant for this work. The simulation was run at $a_{0}=75 \mu \mathrm{m}$ and $a_{0}=$ $40 \mu \mathrm{m}$. This spans typical powder size distributions for LPPBF grade nylon-12. In addition, the simulation was run at $a_{0}=225 \mu \mathrm{m}$ to represent a typical size for powder particles used in rotational molding. Emphasis in the discussion section is given to results from the $40 \mu \mathrm{m}$ simulation as this is the average particle diameter reported by the supplier for PA 250 powder. Results from using 75 and $225 \mu \mathrm{m}$ particle size are shown as comparison to communicate the effect of particle size on coalescence.

\subsection{Printed part density}

The density of printed rectangular prisms was measured in accordance with ASTM D792 using ethanol instead of water as the second fluid, as has been demonstrated as customary for polymer LPPBF parts $[46,47]$. The prisms were nominally $12.5 \times 12.5 \times 15 \mathrm{~mm}$, in the ZX orientation (i.e., with the long axis along the build direction). The $12.5 \times 12.5 \mathrm{~mm}$ square cross-sections in the XY plane were used for collection of transient temperature profiles discussed in Section 3.4. Prisms were brushed to remove excess cake powder. Values reported are the average of three measurements.

\subsection{Tensile testing}

Type IV dogbone samples, fabricated in YX orientation according to print parameters outlined in Section 3.3, were pulled at a constant rate of $50 \mathrm{~mm} \mathrm{~min}^{-1}$ using an Instron 5944 according to ASTM D638. Force and extension data were collected and engineering stress and engineering strain were calculated from the collected data using caliper measurements from the dogbone specimens prior to testing. Reported values are the average of five specimens unless otherwise noted. These results are used to correlate printed part strength with coalescence.

\section{Results and discussion}

The proposed method for determining the CCR is summarized in the following five steps: 1 . Choose LPPBF laser process settings; 2. Obtain transient temperature profiles from each process parameter value combination via IR thermography and heat transfer modeling; 3. Predict physical gelation according to the temperature profile; 4 . Predict coalescence according to the temperature profile; 5 . Compare extent of coalescence to critical coalescence threshold. Parts 1 and 2 are briefly discussed in Section 4.1; however, this text focuses on using the transient temperature profiles to validate the CCR concept in parts 3-5 (Sections 4.2-4.5) and connection to printed part performance (Section 4.6).

\subsection{Mapping laser parameter values to transient temperature profiles}

Maximum temperatures and resulting decay times measured according to Section 3.4 are reported in Table 3. As expected, increasing EMR (via increasing laser power) is observed to increase maximum temperature of the polymer powder bed. One key threshold is the equilibrium melting temperature of nylon- 12 at $466 \mathrm{~K}\left(193^{\circ} \mathrm{C}\right)$. The polymer must be fully melted in order for the surface temperature of the powder to have increased above that temperature. All samples except $\mathrm{EMR}=0.25$ and $\mathrm{EMR}=0.59$ achieve this temperature.

\section{Table 3}

Characteristic values determined from IR thermography of laser scanning nylon- 12 . Values presented are the average of 7 scanned areas over 4 independent trials. Maximum temperature and decay time increase with increasing EMR.

\begin{tabular}{llll}
\hline $\begin{array}{l}\text { Power } \\
{[\mathrm{W}]}\end{array}$ & EMR & $\begin{array}{l}\text { Average maximum } \\
\text { temperature } \\
{[\mathrm{K}]\left(\left[{ }^{\circ} \mathrm{C}\right]\right)}\end{array}$ & $\begin{array}{l}\text { St. dev. of maximum } \\
\text { temperature }\end{array}$ \\
\hline 3 & 0.25 & $439(166)$ & 3.64 \\
7 & 0.59 & $445(172)$ & 2.99 \\
13 & 1.09 & $454(180)$ & 4.79 \\
18 & 1.51 & $460(187)$ & 5.69 \\
25 & 2.09 & $477(203)$ & 5.19 \\
28 & 2.34 & $477(204)$ & 3.44 \\
30 & 2.51 & $480(207)$ & 3.23 \\
35 & 2.93 & $494(221)$ & 3.56 \\
45 & 3.77 & $521(248)$ & 4.05 \\
55 & 4.60 & $538(265)$ & 4.77 \\
\hline
\end{tabular}


(a)

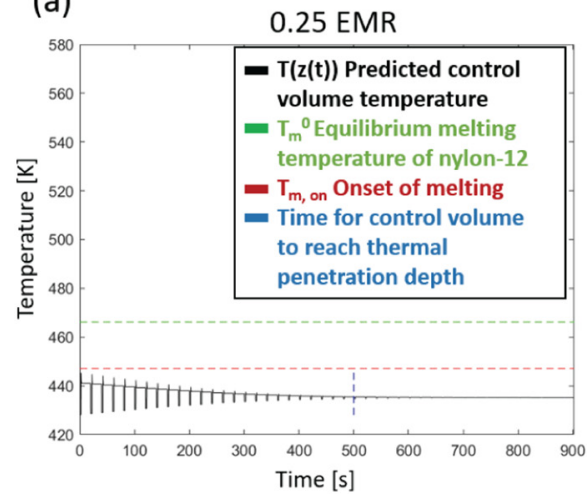

(b)

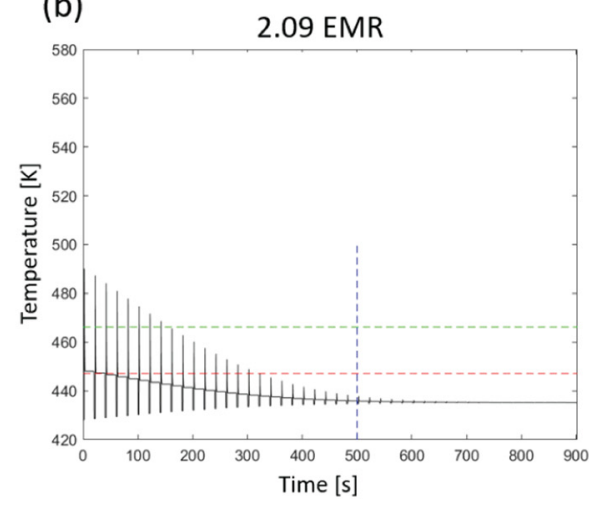

(c)

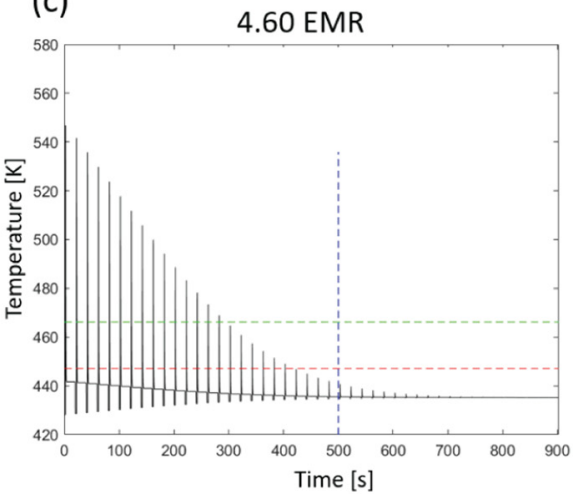

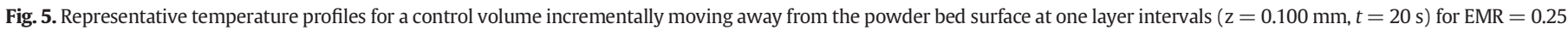

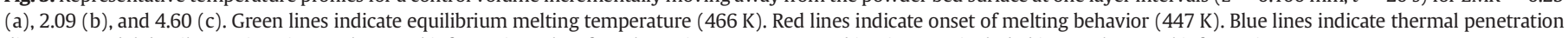
distance. Model details are given in supplemental information. Plots for other print parameter combinations are included in supplemental information.

LPPBF was modeled as a semi-infinite body in order to estimate the influence of additive layering on coalescence. Representative transient temperature profiles from single trials of $0.25,2.09$, and $4.60 \mathrm{EMR}$ are shown in Fig. $5 a-c$. These modeled $T(z, t)$ profiles model a control volume in any given layer incrementally moving away from the powder bed surface. Similar plots for the other process parameter combinations are included in supplemental information. The transient temperature profiles shown in Fig. 5 illustrate the similar, albeit muted, exponential decay at different z-depths as observed for the powder bed surface. As expected, the higher EMR laser parameter combination raises the peak temperature experienced by the control volume above $T_{m}^{0}$ for more layers. Since a temperature above $T_{m}^{0}$ implies complete melting, physical gelation cannot occur until after the control volume ceases to be heated above $T_{m}^{0}$. The temperature never exceeds $T_{m}^{0}$ when using a 0.25 EMR laser parameter combination, exceeds $T_{m}^{0}$ for 8 layers ( $160 \mathrm{~s}$ ) when using 2.09 EMR, and exceeds $T_{m}^{0}$ for 15 layers ( $\left.300 \mathrm{~s}\right)$ when using 4.60 EMR (see Fig. 5). This demonstrates that high EMR laser parameter combinations not only achieve higher temperatures in a single layer, but continue re-heating the polymer control volume above critical melting thresholds for longer. The importance of maintaining a high temperature for a long time to ensure coalescence is further discussed in Sections 4.4 and 4.5 .

\subsection{Simulated two-particle coalescence via UCM model}

The Upper-convected Maxwell (UCM) equation (see equations in supplemental information) was used to simulate $600 \mathrm{~s}$ of two-particle coalescence every degree from 433 to $673 \mathrm{~K}\left(160-400{ }^{\circ} \mathrm{C}\right)$ using published data for LPPBF grade nylon-12 as described and referenced in Section 3.6. Plots of dimensionless neck growth over time at each maximum temperature observed from laser-scanning is presented in Fig. 6a. The dashed horizontal line indicates 0.835 dimensionless neck radius, the theoretical transition between physical regimes of viscous coalescence and bubble diffusion. Critical fusion times $\left(t_{C F}\right)$ for coalescence behavior at all simulated temperatures are plotted in Fig. $6 \mathrm{~b}$ and for the specific temperatures correlated to this study's laser parameters in Table 3. Temperature-dependent neck growth rate (i.e., $\left(\frac{x}{a}\right)^{\prime}$ from Eqs. (3) and (4)) is taken to be $\frac{0.835}{t_{C F}}$ at each temperature.

Increasing coalescence rate with increasing temperature, as shown in Fig. 6a, intuitively correlates with decreasing viscosity at higher temperatures and the role viscosity plays in opposing coalescence (see Eq. (1)). The exponential decay shape of $t_{C F}$ versus temperature in Fig. $6 \mathrm{~b}$ underscores the importance of reaching higher temperatures to ensure rapid coalescence. This behavior alludes to the importance of polymer particles remaining hot and amorphous for as long as possible during LPPBF manufacturing to ensure coalescence. However, the quasiisothermal temperature profile characteristic of LPPBF only allows for $1-2 \mathrm{~s}$ at peak processing temperatures before cooling back toward the chamber temperature; e.g., $434-443 \mathrm{~K}$ for nylon-12. At these temperatures, critical fusion is not reached until $300-450 \mathrm{~s}$ for the $40 \mu \mathrm{m}$ particles, and longer than $600 \mathrm{~s}$ for the larger particles considered. At these longer timescales, it is possible for crystallization to arrest coalescence. The interplay between crystallization and coalescence is further discussed in Section 4.4.

The three initial particle diameters are specifically chosen to show the large impact of particle size on coalescence. Typical LPPBF powder has an average particle diameter on the order of $40-75 \mu \mathrm{m}$ while rotational molding is likely to be at least $225 \mu \mathrm{m}$ or larger. Only at the highest temperatures achieved in this study (4.60 EMR, $552 \mathrm{~K}$ ) do the $t_{C F}$ values approach each other regardless of initial diameter. The 40 and $75 \mu \mathrm{m}$ initial diameters approach equivalence near $500 \mathrm{~K}$. For lower temperatures, the difference between initial particle diameter becomes significant exceeding $8 \mathrm{~min}$ for 40 and $225 \mu \mathrm{m}$ diameters at $466 \mathrm{~K}$, the equilibrium melting temperature of nylon-12. Fig. 6 also show the impact of particle size distribution for any given powder sample, regardless of manufacturing technology. While the d50 (i.e., average) particle size is $62 \mu \mathrm{m}$, the $\mathrm{d} 90$ is just over $300 \mu \mathrm{m}$, as reported in Table 1. This low-biased distribution of particle diameters is typical for LPPBF. The small fraction of large particles will coalesce significantly slower than the majority of particles in the powder bed, thereby resulting in defects. The impact of the large particles in the particle size distribution on performance property variance will be discussed further in Section 4.6.2.

In addition to size, powder shape plays an important role in coalescence during LPPBF. One assumption made when using the UCM equations is initially spherical particles. Published literature routinely acknowledges that commercially available LPPBF powders are more "potato shaped" [48]. Powder shape and size together impact powder spreading and powder packing in the build piston during the LPPBF process [49]. Haeri and coauthors report that particles with an aspect ratio of 1.5 (i.e., non-spherical) affect maximum bed densities under optimized spreading conditions [49,50], and a denser powder bed before laser scanning should increase final part coalescence. However, prior literature has also reported that pairs of non-spherical particles coalesce at a slower rate than what is predicted by assuming perfect spheres for the UCM equations [51]. To incorporate the volumetric effects of powder shape and spreading on coalescence, some form of multi-body (i.e., three of more) coalescence modeling must be used in place of the two-particle coalescence model employed in the present work. 
(a)

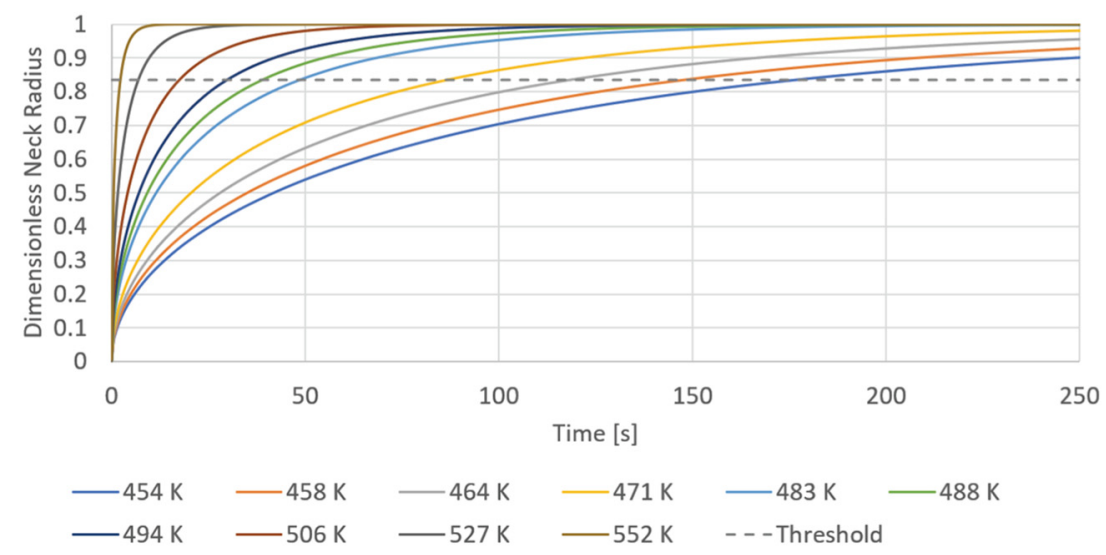

(b)

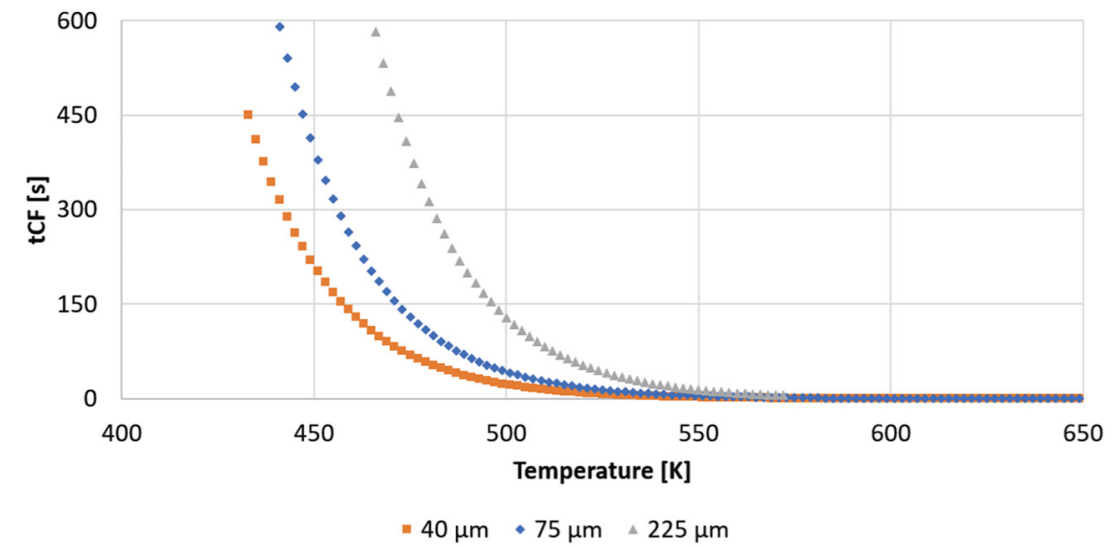

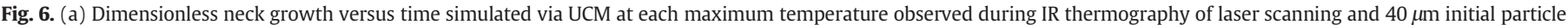

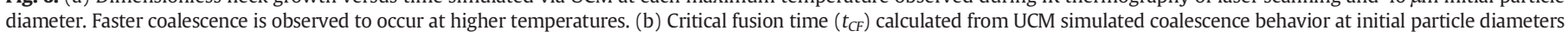
of 40,75 , and $225 \mu \mathrm{m}$.

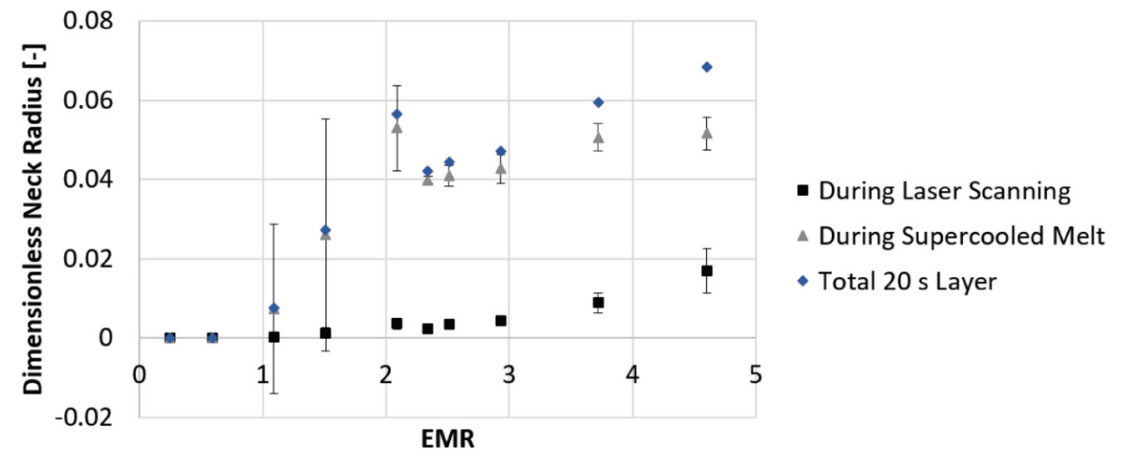

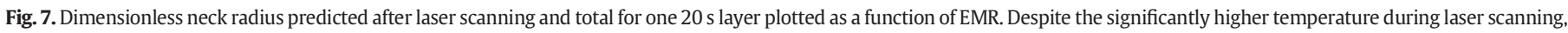
the bulk of neck growth occurs as a supercooled melt.

\subsection{Coalescence within the laser scanning regime}

As described in Section 2.1, predictions of two-particle neck growth during the short-time laser scanning regime is calculated via Eq. (6) as the sum of fractional neck growth based on discretizing the laser decay profile into steps of constant change in temperature (see also Fig. 4). In this work, decay profiles were discretized into single degree ( $1 \mathrm{~K})$ steps. Discretization was performed on each transient temperature profile obtained from IR thermography. The dimensionless neck radii plotted against EMR in Fig. 7 are best fit according to an exponential model. This makes sense for an "activated" process like coalescence [12,29].

Within each layer, the laser-heating timescale is only a few seconds and the polymer exists as a supercooled melt closer to $T_{b \text {, inf }}$ than to $T_{\max }$ for the remaining layer time.

\subsection{Flow arrested by physical gelation}

The degree of supercooling, embodied in $T_{\text {baseline, }}$ is the most influential machine parameter for crystallization kinetics. Crystallization rate 


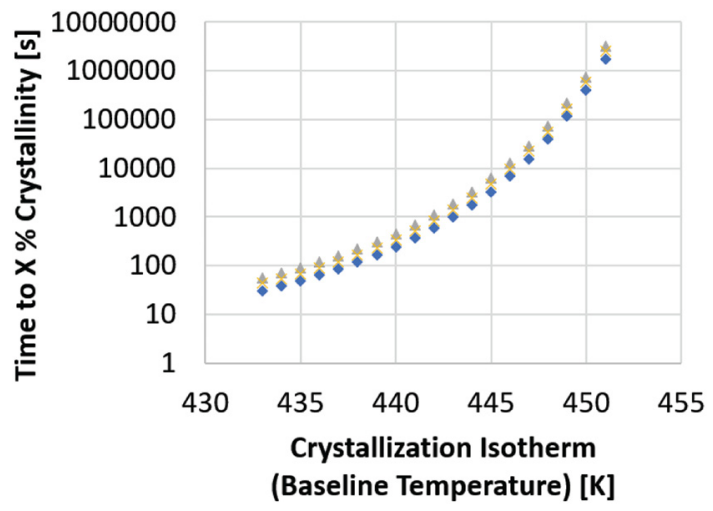

$$
\triangle X=10 \times X=5 \quad X=2
$$

Fig. 8. Time to 2,5 , and $10 \%$ relative crystallinity (estimated by the Avrami equation) as a function of the re-established baseline temperature (measured via IR thermography) and the EMR affecting the baseline temperature. Physical gelation of nylon-12 assumed to occur at $10 \%$ relative crystallinity.

reaches a maximum at some temperature between $T_{g}$ and $T_{m}^{0}$. The value of $T_{\text {baseline }}$ relative to the temperature of maximum crystallization rate determines the value of $t_{A F}$. If $T_{\text {baseline }}$ shifts closer to $T_{m}^{0}$ and away from the temperature of maximum crystallization rate, the rate of crystallization will be slower thereby increasing $t_{A F}$ and promoting particle fusion.

It is intuitive that faster coalescence is achieved with increasing temperature; however, it is less intuitive that the larger magnitude of increase stems from increasing the time to physical gelation (i.e., $t_{A F}$ ) more than decreasing the time to closed pores (i.e., $t_{C F}$ ). Compare the dependence of $t_{C F}$ (Fig. 6b) and time to $10 \%$ crystallinity $\left(t_{X 10}\right)$ (Fig. 8) on temperatures within the range of the control volume in Fig. $5 \mathrm{c}$. The temperatures of note are (1) the temperature of the re-established baseline after the initial laser scan $\left(T_{\text {baseline }}\right),(2)$ the equilibrium temperature of the bulk powder bed isolated from the influence of surface scanning $\left(T_{b, \text { inf }}\right)$, and (3) the rate of decrease from one to the other. For the 4.60 EMR specimen depicted in Fig. $5 c, T_{\text {baseline }}$ is $449 \mathrm{~K}$ $\left(176{ }^{\circ} \mathrm{C}\right)$ and $T_{b \text {, inf }}$ is $433 \mathrm{~K}\left(160{ }^{\circ} \mathrm{C}\right)$. The difference in $t_{C F}$ is $230 \mathrm{~s}$, while the difference in $t_{X 10}$ is $216,490 \mathrm{~s}$ over this temperature range. This emphasizes the two-fold importance of achieving full-melting and having a high bed temperature.

The large absolute numerical value of time to $10 \%$ crystallinity renders this the most influential number in calculating CCR. There are many assumptions made to obtain this number. The primary assumption being that $10 \%$ crystallinity is the onset of physical gelation for LPPBF-grade nylon-12. It is possible that $t_{X 10}$ is too high, and that gelation occurs at a lower percent crystallinity. However, the bigger concern is the precision of measurement of the IR camera is $\pm 2 \mathrm{~K}$, and a $4 \mathrm{~K}$ swing in temperature results in either a difference of $100 \mathrm{~s}$ or 210,000 s depending on the exact temperatures. Similarly, any unaccounted for cooling of the supercooled melt will drastically reduce $t_{X 10}$.
Because samples with EMR $=0.25$ and 0.59 affected average maximum temperatures of less than $T_{m}^{0}$, the $t_{A F \text {, chamber }}$ for these samples will be adjusted to the estimated fraction of crystallinity melted. The fraction melted was estimated using the "running integral" analysis tool in TA Instruments' Universal Analysis (plot included in supplemental information). The entire melt peak was taken to be $100 \%$, and the area fraction at $454 \mathrm{~K}(\mathrm{EMR}=0.25)$ and $458 \mathrm{~K}(\mathrm{EMR}=0.59)$ were found to be 67.6 and $94.7 \%$, respectively. The same "fractional melt" approach is taken when calculating any layers from any sample that do not reach $T_{m}^{0}$.

\subsection{Critical coalescence ratio}

Values for available fusion time $\left(t_{A F}\right)$ and dimensionless neck radius growth inside that time are tabulated in Table 4. Resultant "critical coalescence ratio" of total predicted neck radius at physical gelation to dimensionless neck radius at the transition to closed pore morphology $(0.835)$ is tabulated in Table 4 and plotted against EMR in Fig. 9. Excluding 2.09 EMR, the data take the expected sigmoidal shape. Based on a third-order polynomial fit of reported CCR values (excluding 2.09 EMR, $R^{2}=0.987$ ), the transition to closed pores should occur at 2.16 EMR.

As will be shown in Section 4.6 through the evaluation of the physical properties of the printed parts, process parameter combinations resulting in $\mathrm{CCR}<1$ can form parts that will survive depowdering; however, only print parameter combinations resulting in CCR values above 1 will fabricate parts inside the mechanical property plateau and have the potential for maximum performance properties resulting from densification. CCR at, or slightly above, 1 indicates the most energy efficient manner to fabricate parts of near optimum mechanical performance. The CCR does not replace a traditional statistical design of experiments approach to process parameter optimization, but it does enhance the framework for making engineering decisions and discussing the tradeoffs between manufacturing energy cost and part performance.

Some values for predicted final dimensionless neck radius shown in Table 4 exceed 1 , which is not physically possible as it indicates the contact neck has grown larger than the overall particle radius. This is an artifact of assuming a constant dimensionless neck growth rate for each isothermal temperature without considering the extent of pre-existing dimensionless neck growth. Both Scherer's model [12] and the UCM model (Fig. 6) predict a reduction in coalescence rate as the neck approaches the new radius of the combined and fully coalesced particle. Future work should update the CCR calculation to determine coalescence rate at every point as a function of both temperature and existing neck radius. Although this will improve the accuracy of the CCR calculation and alter the high EMR values, the authors expect only a small deviation from the currently predicted mechanical property plateau threshold where CCR $=1$ at 2.16 EMR.

Table 4

Component and calculated values for critical coalescence ratio (CCR) for measured transient temperature profiles resulting from each tested laser parameter combination. Physical gelation is assumed to be $10 \%$ relative crystallinity for tabulated values.

\begin{tabular}{|c|c|c|c|c|}
\hline Power [W] & EMR & $t_{A F}[\mathrm{~s}]$ & Total Dimensionless Neck Radius Growth [-] & CCR \\
\hline 3 & 0.25 & 0 & $0.00332 \pm 0.0122$ & 0.004 \\
\hline 7 & 0.59 & 0 & $0.0115 \pm 0.0294$ & 0.014 \\
\hline 13 & 1.09 & $508 \pm 12$ & $0.149 \pm 0.428$ & 0.179 \\
\hline 18 & 1.51 & $488 \pm 40$ & $0.562 \pm 0.626$ & 0.673 \\
\hline 25 & 2.09 & $512 \pm 40$ & $1.24 \pm 0.192$ & 1.49 \\
\hline 28 & 2.34 & $457 \pm 18$ & $0.981 \pm 0.046$ & 1.17 \\
\hline 30 & 2.51 & $488 \pm 20$ & $1.07 \pm 0.064$ & 1.28 \\
\hline 35 & 2.93 & $518 \pm 14$ & $1.17 \pm 0.065$ & 1.40 \\
\hline 45 & 3.77 & $581 \pm 13$ & $1.41 \pm 0.053$ & 1.69 \\
\hline 55 & 4.60 & $603 \pm 11$ & $1.52 \pm 0.055$ & 1.81 \\
\hline
\end{tabular}




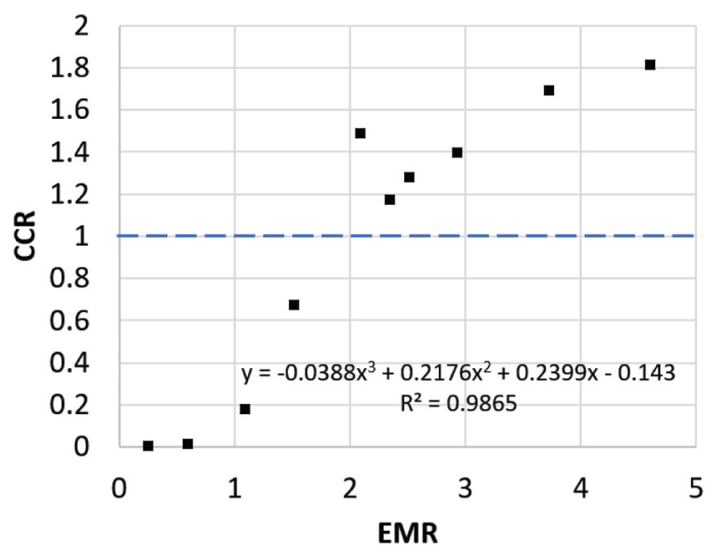

Fig. 9. Proposed "critical coalescence ratio" (CCR) plotted as a function of EMR. Transition to closed pores (i.e., $C C R=1$ ) calculated to occur at 2.16 EMR based on third-order polynomial fit of CCR data excluding the point at 2.09 EMR.

\subsection{CCR correlation to printed part properties}

\subsubsection{Printed density}

Fig. 10 shows the plot of measured relative density of printed $12.5 \mathrm{~mm}$ cubes as a function of EMR. Fully dense is taken to be $1.01 \mathrm{~g}$ $\mathrm{cm}^{-3}$ per manufacturer's specification for PA 250 [52]. Ignoring the outlier at EMR $=0.25$, the observed sigmoidal trend is the one expected from the consolidation models referenced in the introduction (Scherer [12], Tontowi and Childs [29], Fig. 2c). Scherer's threshold for closed pores at 0.94 relative density occurs between EMR $=2.09$ ( 0.928 relative density) and 2.34 ( 0.952 relative density). There is a drastic reduction in the rate of change of density with respect to EMR around this point. The CCR predicted transition (i.e., CCR = 1) at 2.16 EMR falls inside this range.

The outlier at EMR $=0.25$ and the comparatively larger data scatter for samples with EMR 1.09 and below arise from testing near the sensitivity limit for the balance. These samples with the three lowest EMR values were observed to float in ethanol indicating a large amount of trapped air. When soaked in ethanol, samples at EMR $=0.25$ and 1.09 eventually saturated and sunk, indicating surface-connected porosity. The sample at EMR $=0.59$ did not sink therefore necessitating the use of a cage-restraining fixture and a negative value for weight in ethanol. The authors hypothesize the EMR $=0.25$ outlier is a result of a significant amount of porosity that is also connected to the surface, such as may arise from an extremely low degree of powder consolidation. The sample at 0.25 EMR is effectively a lattice. This is supported by the

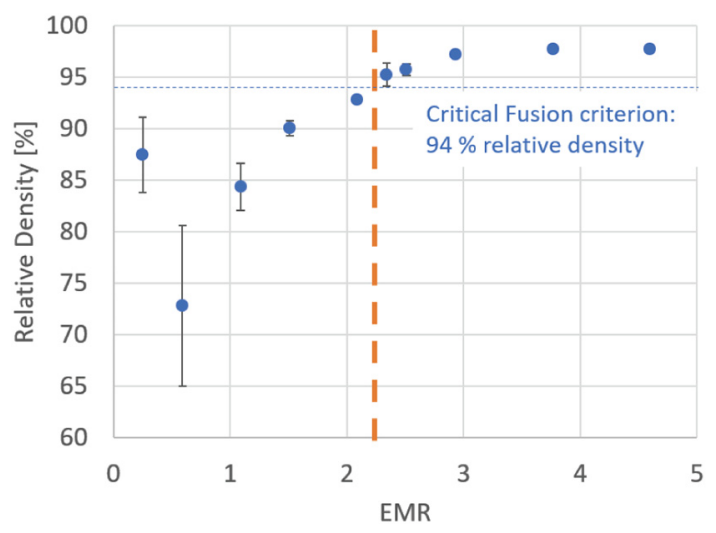

Fig. 10. Relative density of nylon-12 (PA 250) printed parts measured via weight in ethanol/weight in air method. The highlighted region contains the predicted transition to closed pores at 2.16 EMR. measured weight in air for EMR $=0.25$ being less than that of the 0.59 EMR sample $(0.91 \mathrm{~g}<0.97 \mathrm{~g})$ for printed parts of nominally the same volume.

The seminal work by Vasquez and coauthors does not include density or porosity measurements of printed parts as they anticipated no significant difference would be observed in their investigated range of EMR ( 1.7-7.5) [7]. The measurements reported in Fig. 10 support that hypothesis, as most of their range lies above the CCR predicted threshold of 2.16 EMR. Pavan and coauthors report porosity measured by computed tomography for LPPBF-grade nylon-12 printed at surface energy densities ranging from $0.025-0.045 \mathrm{~J} \mathrm{~mm}^{-2}$ (EMR $\sim 3.14-5.65$ based on current work). They report porosities less than $5 \%$ for all surface energy densities studied and, although they do not report $t$-test results, their reported porosity means from several energy densities lie within one standard deviation of each other [10]. These previously published reports corroborate the findings presented here.

\subsubsection{Tensile properties}

Ultimate tensile strength, elongation at break, and Young's modulus for specimens printed at each set of process parameters are shown in Fig. 11. All three tensile properties show two regimes of dependence on EMR (or surface energy density). Although prisms for density measurement were able to be fabricated at the lowest laser parameter combination (0.25 EMR), tensile specimens fabricated at these settings did not survive depowdering and preparation for tensile testing.

The observed onset of performance property plateau occurs at slightly different EMRs for each tensile property. The transition for elongation at break occurs first at 2.09 EMR, then ultimate tensile strength at 2.34 EMR, and finally Young's modulus transitions at 2.51 EMR. All observed transitions are within 0.35 EMR of the CCR predicted transition of 2.16 EMR. Although macro-scale particle consolidation is a major contributing factor to each of these properties, it is not the sole factor. Percent crystallinity, crystalline morphology, and entanglement also contribute to these performance properties in different ways. Therefore, while the CCR predicted transition EMR is close to the observed transition points, it is reasonable for the predicted transition EMR to deviate from real values.

Vasquez and coauthors include second order polynomial fits on their mechanical property plots from the original EMR paper, but do not justify this decision [7]. The authors believe the same data can be interpreted as two linear regimes, using typical consolidation behavior (i.e., viscous coalescence transitioning to bubble diffusion) as the justification for trend shape. Starr and coauthors recognized the transition as a threshold, but offer no explanation for its origins [6].

Fig. 2, which depicts aggregated ultimate and yield tensile strength as a function of surface energy density reported from published literature, was included as part of the introduction section. The observed trend description given by Starr, et al. of an initial steep slope followed by a region where tensile properties are generally independent of energy density [6] served to motivate this work. The observed transition EMR for published ultimate tensile strength values is consistent with the transition EMR observed in this work and predicted by the CCR. Starr and coauthors report the transition in yield strength to occur at $0.012 \mathrm{~J} \mathrm{~mm}^{-2}$ [6], which appears to remain consistent even when including yield strength data from Caulfield [5]. The authors estimate the transition in ultimate tensile strength to be around $0.017 \mathrm{~J} \mathrm{~mm}^{-2}$ based on Fig. 2a. The CCR predicted transition of 2.16 EMR corresponds to an energy density of $0.0172 \mathrm{~J} \mathrm{~mm}^{-2}$, which agrees with their published findings. Starr, et al.'s value for onset of performance property plateau based on Yield Strength of $0.012 \mathrm{~mm}^{-2}$ corresponds to 1.51 EMR and a CCR of 0.58 from the third-order polynomial fit. This indicates that yield strength is sensitive to factors beyond density in a manner that failure properties (e.g., tensile strength and elongation) are not.

For printed part density, ultimate tensile strength, and elongation at break (Figs. 10 and 11), the standard deviation decreases with increasing EMR. This is likely the result of more complete powder melting as 

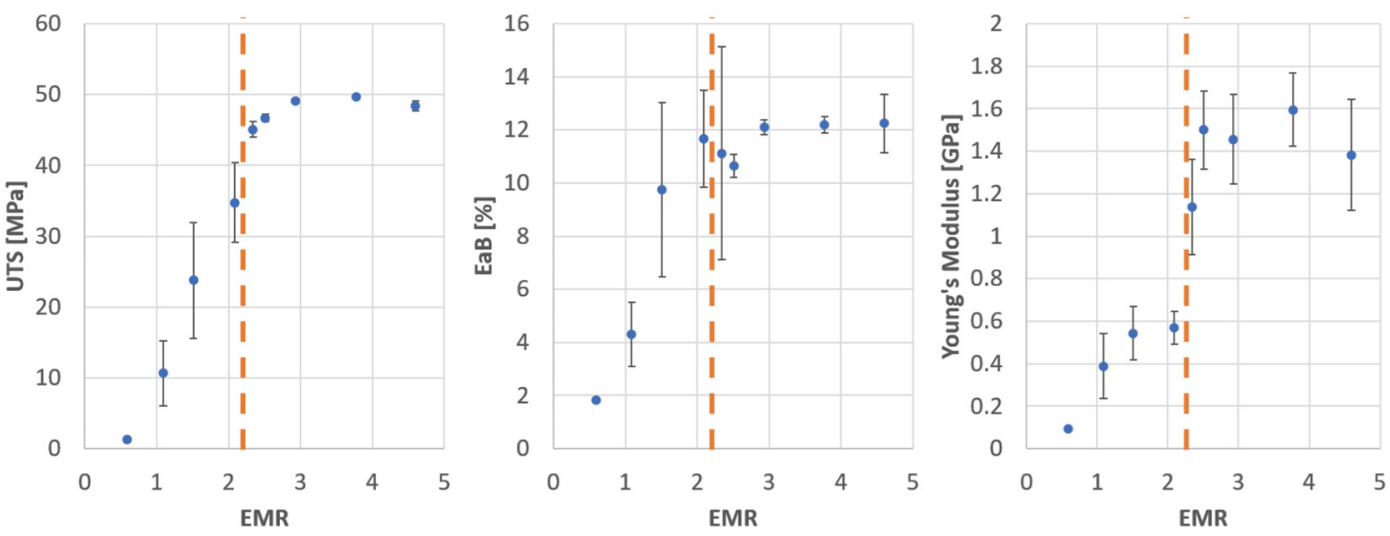

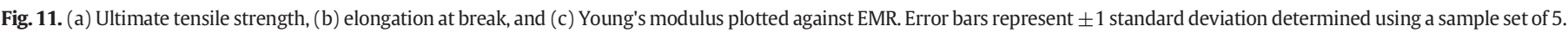
The highlighted regions contains the predicted transition to closed pores at 2.16 EMR (i.e., CCR $=1$ ).

even the particles with diameters at the high end of the size distribution $(\sim 300 \mu \mathrm{m}$; Table 1$)$ are melted on the time scale of processing. This follows the discussion in Section 4.2 where the UCM estimates slower coalescence rates for particles of a larger initial diameter. Since the beam speed remains consistent for all process parameter value combinations in this work, the time of processing also remains consistent; therefore the increased maximum temperature (Table 3 ) at high EMR is able to melt larger particles more completely reducing the number of defects incorporated into printed parts and reducing variation in part performance.

\subsection{Critical analysis of the $C C R$}

This work supports the hypothesis that the change in scaling relationship between mechanical properties and energy density coincides with the transition to bubble diffusion physics at 0.835 dimensionless neck radius. However, it should be noted that the framework is heavily dependent on the accuracy and precision of the thermal camera used to generate transient temperature profiles. Results discussed in Sections 4.1-4.6.2 use the mean of 28 transient temperature measurements. For comparison, the minimum and maximum temperature and CCR values for each corresponding EMR are included in Table 5.

The variability presented in Table 5 results from the combination of machine and measurement variation. The main analysis has been performed on the mean of 28 replicates to reveal the statistically significant polymer-processing relationship trends. This variability may be reduced by choosing a more complex thermal model to represent LPPBF, such as including volumetric heat generation via finite element modeling [53]. Volumetric heat generation is a more realistic representation of LPPBF than the assumption that all heat generation occurs through

Table 5

Minimum and maximum measured powder bed surface temperatures and resultant CCR for each EMR laser parameter combination.

\begin{tabular}{llllll}
\hline $\begin{array}{l}\text { Power } \\
{[\mathrm{W}]}\end{array}$ & EMR & $\begin{array}{l}\text { Minimum } \\
\text { measured peak } \\
\text { temperature } \\
{[\mathrm{K}]\left(\left[{ }^{\circ} \mathrm{C}\right]\right)}\end{array}$ & $\begin{array}{l}\text { Minimum } \\
\mathrm{CCR}\end{array}$ & $\begin{array}{l}\text { Maximum } \\
\text { measured peak } \\
\text { temperature } \\
{[\mathrm{K}]\left(\left[{ }^{\circ} \mathrm{C}\right]\right)}\end{array}$ & $\begin{array}{l}\text { Maximum } \\
\mathrm{CCR}\end{array}$ \\
\hline 3 & 0.25 & $434(161)$ & 0 & $447(174)$ & 0.06 \\
7 & 0.59 & $440(167)$ & 0 & $451(178)$ & 0.16 \\
13 & 1.09 & $446(173)$ & 0 & $462(189)$ & 1.66 \\
18 & 1.51 & $452(179)$ & 0 & $473(200)$ & 1.75 \\
25 & 2.09 & $469(196)$ & 1.21 & $490(217)$ & 1.91 \\
28 & 2.34 & $471(198)$ & 1.24 & $485(212)$ & 1.32 \\
30 & 2.51 & $474(201)$ & 1.24 & $485(212)$ & 1.45 \\
35 & 2.93 & $486(213)$ & 1.30 & $502(229)$ & 1.56 \\
45 & 3.77 & $511(238)$ & 1.59 & $529(256)$ & 1.81 \\
55 & 4.60 & $530(257)$ & 1.77 & $554(281)$ & 1.94 \\
\hline
\end{tabular}

laser-polymer interactions at the powder bed surface and that the bulk powder temperature remains constant throughout the build. These assumptions become less valid for a build densely packed with parts where the amount of input energy can greatly outpace thermal diffusion in the powder bed increasing the overall bulk powder temperature and not simply the local temperature increase discussed in Section 2.2.

One aspect of caution observed in the 'minimum' columns in Table 5 is the minimum peak temperature for the samples 1.09 and 1.51 EMR now fall below the end-of-melt temperature $463 \mathrm{~K}\left(190{ }^{\circ} \mathrm{C}\right)$ resulting in CCRs of zero due to incomplete melting. For this work, the degree of melting has been assumed to be the area percent of the DSC melting endotherm up to the average peak temperature affected by each laser setting combination. The CCR framework currently makes an overly simplistic assumption that the fractional melt behaves identically to a complete melt. This is not likely to be accurate based on published investigations into partial melting in LPPBF by Zarringhalam, et al. [2,3]. Crystallization behavior from such partial melting would decrease the time to physical gelation, especially if melting occurred in the core/ shell manner proposed by Zarringhalam, et al. [2]. Crystallization from core/shell melting renders the bulk, simultaneous, and unimpinged nucleation and growth assumptions of the Avrami equations less valid. Rigorous verification of complete melting in a one-layer thick control volume should include melting kinetics based on in situ $\mathrm{x}$-ray scattering. Similarly, results from the UCM-model are more reliable at temperatures above $T_{m}^{0}$ where the control volume is fully molten.

The current form of the CCR only characterizes the polymer response in terms of two-particle neck growth rate. This can be improved in a variety of ways. Multi-particle modeling efforts, including the Farz factor [54], should more accurately estimate the time to reach 0.94 relative density. The timescale of polymer response response should be expanded to include the kinetics of melting and JKR theory for initial elastic neck formation between particles as a prelude to viscous coalescence. Such work follows a different set of governing physics, as has been discussed by Jagota, et al. [55]. Work by Eggers, et al. [56] and Benedetti, et al. [57] have demonstrated the importance of the initial period of particle contact $\left(r_{\text {neck }} \leq 0.1\right)$ on the rate and extent of fusion. Their work is based on the Stokes equations instead of UCM, and indicates the actual shape, and therefore area, of the contact area between two particles resembles a toroid instead of the simple curvature assumed by the Bellehumuer, et al. and Scribben, et al. models used in the present work. Expanding the CCR framework to include detailed information regarding the initial stages of coalescence would improve accuracy and likely narrow data scatter especially of low laser energy, low surface temperature parameter combinations. 


\section{Conclusions}

This work focused on understanding the relationship between transient temperature profiles produced by the print settings on the fusion response of nylon-12 powder in most prevalent form of polymer powder bed fusion (PBF), laser polymer powder bed fusion (LPPBF). This was accomplished through predicting the extent of coalescence using the Upper-convected Maxwell equations and physical gelation using Lauritzen-Hoffman and Avrami equations. The predicted extent of coalescence was compared against the theoretical transition in consolidation physics at $94 \%$ relative density (i.e., 0.835 dimensionless neck radius) to calculate the "critical coalescence ratio" (CCR) for ten combinations of laser parameter values. Parameter value combinations achieving a CCR greater than 1 were observed to print parts with mechanical properties inside the plateau region and greater than $94 \%$ relative density, just as hypothesized. The reported results support use of the CCR as a method for validating laser parameter value combinations to achieve parts of maximum performance.

The threshold where CCR $=1$ at 2.16 EMR was found to accurately predict $94 \%$ relative density in printed parts for nylon- 12 at given processing conditions. The predicted threshold of 2.16 EMR falls in between the studied process parameter combinations of 2.09 EMR, which was found to produce parts of 0.928 relative density, and 2.34 EMR, which was found to produce parts of 0.952 relative density. This same transition window between 2.09 and 2.34 EMR was observed to coincide with the onset of performance property plateau for ultimate tensile strength and elongation at break for nylon-12 printed at the chosen parameter combinations. The observed onset of performance property plateau was also found to agree with data published in prior literature. This trend is hypothesized to be consistent for any performance property dictated by density; however, the value of 2.16 EMR is specific to nylon-12. Major contributing factors to the CCR calculation, such as thermal response to laser irradiation, viscoelastic properties, and crystallization kinetics, are highly dependent on polymer chemical composition and architecture. The major contribution of this manuscript is outlining the framework and methodology for calculating the CCR for any polymer.

Discussion of the CCR concept in this manuscript addresses shortcomings in the energy melt ratio's (EMR) framework for process parameter value selection based solely on polymer melting behavior. Namely, CCR aids in addressing why viable parts are not generated at $\mathrm{EMR}=1$, and why there is an increasing trend in mechanical properties followed by a plateau region with increasing EMR prior to $\mathrm{EMR}_{\text {deg }}$. The foundational theory to the CCR provides a connection to quantify the polymer's response to imposed laser heating. Providing energy to melt the polymer is required, but additional energy to overcome barriers to coalescence (e.g., biaxial extensional viscosity) is also required.

Choosing the CCR to be a ratio of characteristic timescales emphasizes the time-dependent nature of viscoelastic coalescence. The same energy density delivered through multiple combinations of component parameters has been shown to affect different structures and properties of printed parts. It is expected that the transient temperature profiles used to determine the CCR will show differences in equivalent energy densities obtained by varying more than laser power. Future work should validate this hypothesis. This being said, time-independent energy barriers to fusion, such as latent heat of melting and activation energy for flow, are important factors to consider when selecting print settings. In this way, the CCR framework complements the EMR.

All mechanical properties are negatively effected by defects, such as porosity, however, they are each positively effected by different complementary polymer characteristics. Future work should investigate the sensitivity of different mechanical properties to coalescence relative to other factors, like crystallinity. For bulk molding techniques, tensile strength and modulus have been shown to positively scale with increasing crystallinity, while elongation tends to negatively scale with crystallinity [58]. These properties will scale with density to an extent, however, each mechanical property may approach bulk density at a different CCR. The CCR approach would benefit from including the time for chain re-entanlgement, such as proposed by Baird, et al. [59], and implemented for material extrusion additive manufacturing by Gilmer, et al. [60].

\section{Data availability}

Portions of the raw data required to reproduce these findings are available to download from [INSERT PERMANENT WEB LINK(s)]. The processed data required to reproduce these findings are available to download from [INSERT PERMANENT WEB LINK(s)]. Portions of the raw/processed data required to reproduce these findings cannot be shared at this time due to technical or time limitations.

\section{Author statement}

Camden Chatham: Conceptualization, Methodology, Experimentation, MATLAB code, Writing - original draft preparation. Michael Bortner: Conceptualization (coalescence model), Funding, Writing Reviewing and editing. Blake Johnson: Conceptualization (thermal model), Writing - Reviewing and editing. Timothy Long: Supervision, Writing - Reviewing and editing. Christopher Williams: Supervision, Conceptualization, Funding, Writing - Reviewing and Editing.

\section{Declaration of Competing Interest}

None

\section{Acknowledgments}

This work is funded by the Department of Energy's Kansas City National Security Campus, operated by Honeywell Federal Manufacturing \& Technologies, LLC under contract number DE-NA0002839.

The authors would like to acknowledge Garrett Godshall for his assistance in experimentation and helpful discussion regarding twoparticle coalescence, Ian Ho for assistance in infrared thermography measurements, as well as Jackson Bryant and Julia Pimentel for their assistance with powder property measurements. In addition, the authors acknowledge the Macromolecules Innovation Institute (MII) at Virginia Tech for providing a collaborative infrastructure focused across the spectrum of topics in cutting-edge polymer science and engineering research.

\section{Appendix A. Supplementary data}

Supplementary data to this article can be found online at https://doi. org/10.1016/j.matdes.2021.109474.

\section{References}

[1] ASTM, ISO, ISO/ASTM 52900:2015(E), https://www-astm-org.ezproxy.lib.vt.edu/ cgi-bin/resolver.cgi?ISOASTM52900 2015.

[2] H. Zarringhalam, N. Hopkinson, N. Kamperman, J. de Vlieger, Effects of processing on microstructure and properties of SLS Nylon 12, Mater. Sci. Eng. A 435-436 (2006) 172-180, https://doi.org/10.1016/j.msea.2006.07.084.

[3] H. Zarringhalam, C. Majewski, N. Hopkinson, Degree of particle melt in Nylonâ€ 12 selective laser-sintered parts, Rapid Prototyp. J. 15 (2) (2009) 126-132.

[4] M.M. Savalani, L. Hao, P.M. Dickens, Y. Zhang, K.E. Tanner, R.A. Harris, The effects and interactions of fabrication parameters on the properties of selective laser sintered hydroxyapatite polyamide composite biomaterials, Rapid Prototyp. J. 18 (1) (2012) 16-27, https://doi.org/10.1108/13552541211193467.

[5] B. Caulfield, P.E. McHugh, S. Lohfeld, Dependence of mechanical properties of polyamide components on build parameters in the SLS process, J. Mater. Process. Technol. 182 (1-3) (2007) 477-488, https://doi.org/10.1016/j.jmatprotec.2006.09. 007. 
[6] T.L. Starr, T.J. Gornet, J.S. Usher, The effect of process conditions on mechanical properties of laser-sintered nylon, Rapid Prototyp. J. 17 (6) (2011) 418-423, https://doi. org/10.1108/13552541111184143.

[7] M. Vasquez, B. Haworth, N. Hopkinson, Methods for quantifying the stable sintering region in laser sintered Polyamide-12, Polym. Eng. Sci. 53 (6) (2013) 1230-1240, https://doi.org/10.1002/pen23386.

[8] S. Dupin, Etude fondamentale de la transformation du polyamide 12 par frittage laser : mécanismes physico-chimiques et relations microstructures / propriétés Résumé, Ph.D. thesis 2012.

[9] L.V. Milisits, Methodology for Mechanical Property Optimization of Selective Laser Sintered Parts Using Design of Experiments, Ph.D. thesis University of Texas at Austin, 2017.

[10] M. Pavan, M. Faes, D. Strobbe, B. Van Hooreweder, T. Craeghs, D. Moens, W. Dewulf, On the influence of inter-layer time and energy density on selected critical-toquality properties of PA12 parts produced via laser sintering, Polymer Testing 61 (2017) 386-395.

[11] Y.Y. Lin, C.Y. Hui, A. Jagota, The role of viscoelastic adhesive contact in the sintering of polymeric particles, J. Colloid Interface Sci. 237 (2) (2001) 267-282, https://doi. org/10.1006/jcis.2001.7470.

[12] G.W. Scherer, Viscous sintering of a bimodal pore-size distribution, J. Am. Ceram. Soc. $64(11,1984) 709-715$.

[13] C.A. Chatham, T.E. Long, C.B. Williams, Powder bed fusion of poly(phenylene sulfide) at bed temperatures significantly below melting, Additive Manufacturing 28 (2019) 506-516 March.

[14] M. Kamyabi, R. Sotudeh-Gharebagh, R. Zarghami, K. Saleh, Principles of viscous sintering in amorphous powders: a critical review, Chem. Eng. Res. Design 125 (2017) 328-347.

[15] M. Kontopoulou, J. Vlachopoulos, Bubble dissolution in molten polymers and its role in rotational molding, Polym. Eng. Sci. 39 (7) (1999) 1189-1198, https://doi.org/10. 1002/pen.11505

[16] H.B. Ly, E. Monteiro, M. Dal, G. Regnier, On the factors affecting porosity dissolution in selective laser sintering process, AIP Conference Proceedings 1960 (May 2018) (2018)https://doi.org/10.1063/1.5034982.

[17] H.B. Ly, E. Monteiro, T.T. Le, V.M. Le, M. Dal, G. Regnier, B.T. Pham, Prediction and sensitivity analysis of bubble dissolution time in 3D selective laser sintering using ensemble decision trees, Materials 12 (9) (2019)https://doi.org/10.3390/ ma12091544.

[18] B. Haworth, N. Hopkinson, D. Hitt, X. Zhong, Shear viscosity measurements on Polyamide-12 polymers for laser sintering, Rapid Prototyp. J. 19 (1) (2013) 28-36, https://doi.org/10.1108/13552541311292709.

[19] M. Vasquez, B. Haworth, N. Hopkinson, Optimum sintering region for laser sintered nylon-12, Proc. Inst. Mech. Eng. B J. Eng. Manuf. 225 (12) (2011) 2240-2248, https://doi.org/10.1177/0954405411414994.

[20] R.J. Crawford, J.L. Throne, Rotational Molding Technology, 1st edition William Andrew, Nowich, NY, 2001.

[21] J.J. Frenkel, Viscous flow of crystalline bodies under the action of surface tension, J. Phys. 9 (1945) 385.

[22] C. Bellehumeur, Polymer Sintering and its Role in Rotational Molding, Phd McMaster University, 1997.

[23] E. Scribben, D. Baird, P. Wapperom, The role of transient rheology in polymeric sintering, Rheol. Acta 45 (6) (2006) 825-839, https://doi.org/10.1007/s00397005-0039-1.

[24] R. Goodridge, C. Tuck, R. Hague, Laser sintering of polyamides and other polymers, Prog. Mater. Sci. 57 (2) (2011) 229-267, https://doi.org/10.1016/j.pmatsci.2011. 04.001.

[25] C.T. Bellehumeur, M.K. Bisaria, J. Vlachopoulos, An experimental study and model assessment of polymer sintering, Polym. Eng. Sci. 36 (17) (1996) 2198-2207, https://doi.org/10.1002/pen.10617.

[26] C.T. Bellehumeur, M. Kontopoulou, J. Vlachopoulos, The role of viscoelasticity in polymer sintering, Rheol. Acta 37 (3) (1998) 270-278, https://doi.org/10.1007/ s003970050114.

[27] A. Mokrane, M. Boutaous, S. Xin, Numerical analysis of the heating phase and densification mechanism in polymers selective laser melting process, AIP Conference Proceedings 1960 (May) (2018)https://doi.org/10.1063/1.5035005.

[28] H. Münstedt, Extensional rheology and processing of polymeric materials, Int. Polym. Process. 33 (5) (2018) 594-618, https://doi.org/10.3139/217.3532.

[29] A.E. Tontowi, T. Childs, Density prediction of crystalline polymer sintered parts at various powder bed temperatures, Rapid Prototyp. J. 7 (3) (2001) 180-184, https://doi.org/10.1108/13552540110395637.

[30] M. Sun, J. Nelson, J. Beaman, J. Barlow, A model for partial viscous sintering, Solid Freeform Fabrication Symposium Proceedings 1991, pp. 46-55.

[31] K. Wudy, M. Drexler, L. Lanzl, D. Drummer, Analysis of time dependent thermal properties for high rates in selective laser sintering, Rapid Prototyp. J. 24 (5) (2018) 894-900, https://doi.org/10.1108/RPJ-01-2017-0013.

[32] K. Wudy, S. Greiner, M. Zhao, D. Drummer, Selective laser beam melting of polymers: in situ and offline measurements for process adapted thermal characterization, Procedia CIRP 74 (2018) 238-243, https://doi.org/10.1016/j.procir.2018.08. 102.

[33] R.M. Patel, J.E. Spruiell, Crystallization kinetics during polymer processing-analysis of available approaches for process modeling, Polym. Eng. Sci. $31(10,1991)$ 730-738, https://doi.org/10.1002/pen.760311008.
[34] A. Ziabicki, The mechanisms of 'neck-like' deformation in high-speed melt spinning 2. Effects of polymer crystallization, J. Non-Newtonian Fluid Mech. 30 (2-3) (1988) 157-168, https://doi.org/10.1016/0377-0257(88)85022-5.

[35] Y.G. Zhou, W.B. Wu, G.Y. Lu, J. Zou, Isothermal and non-isothermal crystallization kinetics and predictive modeling in the solidification of poly(cyclohexylene dimethylene cyclohexanedicarboxylate) melt, J. Elastomers Plastics 49 (2) (2017) 132-156, https://doi.org/10.1177/0095244316641327.

[36] C.A. Chatham, Property-Process-Property Relationships in Powder Bed Fusion Additive Manufacturing of Poly(Phenylene Sulfide): A Case Study toward Predicting Printability from Polymer Properties, Doctor of Philosophy Virginia Polytechnic Institute and State University, 2020.

[37] E.D. Bain, Polymer Powder Bed Fusion Additive Manufacturing: Recent Developments in Materials, Processes, and Applications, ACS Symposium Series, Vol. 1315, American Chemical Society 2019, pp. 7-36, https://doi.org/10.1021/bk-2019-1315. ch002.

[38] M. Zhao, K. Wudy, D. Drummer, Crystallization kinetics of polyamide 12 during selective laser sintering, Polymers 10 (2) (2018) 168, https://doi.org/10.3390/ polym10020168.

[39] T. Hirayama, T. Uneyama, Y. Masubuchi, Characterization of critical gel state of polyamides by viscoelastic, thermal, and IR measurements, Rheol. Acta (2019) 281-290, https://doi.org/10.1007/s00397-019-01136-0.

[40] S. Dupin, O. Lame, C. Barrès, J.-Y. Charmeau, Microstructural origin of physical and mechanical properties of polyamide 12 processed by laser sintering, Eur. Polym. J. 48 (9) (2012) 1611-1621, https://doi.org/10.1016/j.eurpolymj.2012.06.007.

[41] J.A. Lewis, B.G. Compton, J.R. Raney, T.J. Ober, Three-dimensional printed composite structure and 3D printable composite ink formulation, 2016.

[42] A. Lewis, N. Katta, A. Mcelroy, T. Milner, S. Fish, J. Beaman, Investigation of optical coherence tomography imaging in, Solid Freeform Fabrication Symposium (2017) 2725-2736.

[43] ASTM D1895-96, Standard Test Methods for Apparent Density, Bulk Factor, and Pourability of Plastic, Annual Book of ASTM Standards 08 (Reapproved) 2010 1-5, https://doi.org/10.1520/D1895-96R10E01.

[44] World Health Organization, Bulk density and tapped density of powders, QAS 11.450 - The International Pharmacopeia, March 2012 1-6, https://doi.org/10. 1007/s13398-014-0173-7.2.

[45] O. Pokluda, C.T. Bellehumeur, J. Machopoulos, Modification of Frenkel 's Model for sintering, AICHE J. 43 (12) (1997) 3253-3256, https://doi.org/10.1002/aic. 690431213

[46] M. Drexler, M. Lexow, D. Drummer, Selective laser melting of polymer powder part mechanics as function of exposure speed, Phys. Procedia 78 (2015) 328-336 https://doi.org/10.1016/j.phpro.2015.11.047http://www.sciencedirect.com/science/article/pii/S1875389215015370.

[47] M.M. Lexow, M. Drexler, D. Drummer, Fundamental investigation of part properties at accelerated beam speeds in the selective laser sintering process, Rapid Prototyp. J. 23 (6) (2017) 1099-1106, https://doi.org/10.1108/RPJ-04-2016-0060.

[48] C.A. Chatham, T.E. Long, C.B. Williams, A review of the process physics and materia screening methods for polymer powder bed fusion additive manufacturing, Prog. Polym. Sci. 93 (2019) 68-95, https://doi.org/10.1016/j.progpolymsci.2019.03.003.

[49] S. Haeri, Y. Wang, O. Ghita, J. Sun, Discrete element simulation and experimental study of powder spreading process in additive manufacturing, Powder Technology 306 (2017) 45-54, https://doi.org/10.1016/j.powtec.2016.11.002.

[50] S. Haeri, Optimisation of blade type spreaders for powder bed preparation in additive manufacturing using DEM simulations, Powder Technology 321 (2017) 94-104, https://doi.org/10.1016/j.powtec.2017.08.011.

[51] S. Haeri, L. Benedetti, O. Ghita, Effects of particle elongation on the binary coalescence dynamics of powder grains for laser sintering applications, Powder Technology 363 (2020) 245-255, https://doi.org/10.1016/j.powtec.2019.12.025.

[52] L. Advanced Laser Materials, PA 250 Technical Data Sheet, 2011 URL file://G:/ MyDrive/!DREAMSFileStream/Chatham,Camden/Literature/Summer2020/PA250\{ \}specsheet.pdf.

[53] A. Mokrane, M. Boutaous, S. Xin, Process of selective laser sintering of polymer powders: modeling, simulation, and validation, Comptes Rendus - Mecanique 346 (11) (2018) 1087-1103, https://doi.org/10.1016/j.crme.2018.08.002.

[54] A. Hamidi, S. Farzaneh, F. Nony, Z Ortega, S. Khelladi, M. Monzon, F. Bakir, A Tcharkhtchi, Modelling of sintering during rotational moulding of the thermoplastic polymers, Int. J. Mater. Forming 9 (4) (2016) 519-530, https://doi.org/10.1007/ s12289-015-1239-6.

[55] A. Jagota, C. Argento, S. Mazur, Growth of adhesive contacts for Maxwell viscoelastic spheres, J. Appl. Phys. 83 (1) (1998) 250-259, https://doi.org/10.1063/1.366679.

[56] J. Eggers, J.R. Lister, H.A. Stone, Coalescence of liquid drops, J. Fluid Mech. 401 (1999) 293-310.

[57] L. Benedetti, B. Brulé, N. Decraemer, K. Evans, O. Ghita, Evaluation of particle coalescence and its implications in laser sintering, Powder Technol. 342 (2019) 917-928.

[58] P.C. Painter, M.M. Coleman, Essentials of Polymer Science and Engineering, DEStech Publications, Inc, Lancaster, PA, 2009.

[59] R. Pisipati, D. Baird, Correlation of non-linear rheological properties of polymer melts with weld-line strength, Polymer Processing and Properties, Springer 1984, pp. 215-228.

[60] E.L. Gilmer, C. Mansfield, J.M. Gardner, E.J. Siochi, D.G. Baird, M.J. Bortner, Characterization and analysis of Polyetherimide: realizing practical challenges of modeling the extrusion-based additive manufacturing process, ACS Symp. Ser. 1315 (2019) 69-84, https://doi.org/10.1021/bk-2019-1315.ch005. 